\title{
Rotating nonuniform black string solutions
}

\author{
Burkhard Kleihaus ${ }^{1 *}$ Jutta Kunz ${ }^{1 \dagger}$ and Eugen Radu ${ }^{2 \ddagger}$ \\ ${ }^{1}$ Institut für Physik, Universität Oldenburg, Postfach 2503 D-26111 Oldenburg, Germany \\ ${ }^{2}$ Department of Mathematical Physics, National University of Ireland, Maynooth, Ireland
}

\begin{abstract}
We explore via linearized perturbation theory the Gregory-Laflamme instability of rotating black strings with equal magnitude angular momenta. Our results indicate that the Gregory-Laflamme instability persists up to extremality for all even dimensions between six and fourteen. We construct rotating nonuniform black strings with two equal magnitude angular momenta in six dimensions. We see a first indication for the occurrence of a topology changing transition, associated with such rotating nonuniform black strings. Charged nonuniform black string configurations in heterotic string theory are also constructed by employing a solution generation technique.
\end{abstract}

KEYWORDS: black strings, numerical solutions, rotating spacetimes.

\footnotetext{
${ }^{*}$ E-mail: kleihaus@theorie.physik.uni-oldenburg.de

${ }^{\dagger}$ E-mail: kunz@theorie.physik.uni-oldenburg.de

${ }^{\ddagger}$ E-mail: radu@thphys.nuim.ie
} 


\section{Contents}

1. Introduction 1

2. General ansatz and properties of the solutions 3

2.1 The equations 3

2.2 General properties 6

3. Rotating UBS with equal angular momenta 9

3.1 Thermodynamics 9

3.2 Gregory-Laflamme instability 13

4. $D=6$ rotating nonuniform black string solutions 16

4.1 Numerical procedure 16

4.2 Properties of rotating black strings 19

4.2.1 The horizon 19

4.2 .2 A critical temperature 20

4.2 .3 Global charges 23

4.2.4 The ergoregion 25

5. Rotating NUBS in heterotic string theory 28

6. Further remarks. Conclusions. 31

\section{Introduction}

In recent years interest in the properties of gravity in more than $D=4$ dimensions increased significantly. This interest was enhanced by the development of string theory, which requires a ten-dimensional spacetime, to be consistent from a quantum point of view. In order not to contradict observational evidence, the extra dimensions are usually supposed to be compactified on small scales.

Black string solutions, present for $D \geq 5$ spacetime dimensions, are of particular interest, since they exhibit new features that have no analogue in the black hole case. The simplest vacuum static solutions of this type are found by trivially extending to $D$ dimensions the vacuum black hole solutions to Einstein equations in $D-1$ dimensions. These then correspond to uniform black strings (UBS) with horizon topology $S^{D-3} \times S^{1}$.

One of the first steps towards understanding the higher-dimensional solutions is to investigate their classical stability against small perturbations. In a surprising development, 
Gregory and Laflamme (GL) showed that the static UBS solutions are unstable below a critical value of the mass [1]. Following this discovery, a branch of nonuniform black string (NUBS) solutions was found perturbatively from the critical GL string in five [2], six [3] and in higher, up to sixteen, dimensions [4]. This nonuniform branch was numerically extended into the full nonlinear regime for $D=5$ [5] and $D=6$ [3, 5]. In recent work [6] the nonuniform branch was extended for all dimensions up to eleven. These NUBS are static configurations with a nontrivial dependence on the extra dimension, and their mass is always greater than that of the critical UBS (see [7] and also [8] for a recent review of this subject).

Apart from the black string solutions, Kaluza-Klein (KK) theory possesses also a branch of black hole solutions with an event horizon of topology $S^{D-2}$. The numerical results presented in [9, 5] (following a conjecture put forward in [10]) suggest that the black hole branch and the nonuniform string branch merge at a topology changing transition. The problem is also interesting as it is connected by holography to the phase structure of large $N_{c}$ superYang-Mills theory at strong coupling, compactified on a circle (see e.g. [11], [12], 13] ).

Recently, interest in the properties of rotating solutions in more than $D=4$ dimensions increased significantly, as well. Rotating black objects typically exhibit much richer dynamics than their static counterparts, especially in more than four dimensions. A famous example is the black ring solution [14] in five-dimensional vacuum gravity, which has horizon topology $S^{2} \times S^{1}$, its tension and self gravitational attraction being balanced by the rotation of the ring.

The KK theory presents also spinning configurations. The simplest rotating UBS configurations are found by taking the direct product of a $(D-1)$-dimensional Myers-Perry (MP) solution [15] with a circle. These solutions are likely to exhibit a classical GL instability, as well, at least for some range of the parameters. However, previous investigations have focused on static black strings, and no attention has been given to spin. A major obstacle in this direction is that the analytic theory of perturbations of higher-dimensional black holes has not been fully developed yet (see, however, the recent work 16]).

A MP spinning black string in $D$ dimensions is characterized by the mass-energy, the tension, and $[(D-2) / 2]$ angular momenta, where $[(D-2) / 2]$ denotes the integer part of $(D-2) / 2$. The generic rotating nonuniform solutions possess a nontrivial dependence on $(D-4) / 2$ angular coordinates, which therefore pose a difficult numerical problem. In the even-dimensional case, however, the problem can be greatly simplified, when the a priori independent $(D-2) / 2$ angular momenta are chosen to have equal magnitude, since this factorizes the angular dependence [17]. The problem then reduces to studying the solutions of a set of five partial differential equations with dependence only on the radial variable $r$ and the extra dimension $z$.

In this paper we focus on this particular case, by studying first the GL instability of MP UBSs with equal magnitude angular momenta in even spacetime dimensions. These particular MP solutions possess interesting features, which strongly contrast with those of MP solutions with a single nonzero angular momentum. In particular, we note the existence of an upper bound for the scaled angular momenta $J / M^{(D-3) /(D-4)}$ [15, 18], an extremal 
solution being found, when this limit is approached; whereas no such upper bound is present for single angular momentum MP solutions, unless $D=4+1$ or $D=5+1$.

Although the GL instability is inevitable for static vacuum black strings, the presence of rotation (or a gauge field charge) might prevent black strings from exhibiting such an instability. However, our numerical results indicate that the GL instability persists for rotating vacuum black strings all the way to extremality, at least for all (even) dimensions between six and fourteen.

This type of solutions also provides a new laboratory to test the Gubser-Mitra (GM) conjecture [19], that correlates the dynamical and thermodynamical stability for systems with translational symmetry and infinite extent. In this conjecture, the appearance of a negative specific heat of a black string is related to the onset of a classical instability (see [20, 21] for a discussion of this issue in the case of charged static black strings and black $p$-branes). The analysis of the thermodynamical stability of the MP UBS indicates that thermodynamical stability becomes possible when taking a canonical ensemble, for solutions near extremality. However, in a grand canonical ensemble all UBS configurations are unstable, which agrees with the results we found when studying the GL instability.

In $D=6$ we constructed the set of rotating nonuniform black strings numerically. These rotating NUBS solutions can be found by starting with static NUBS configurations and increasing the value of angular velocity of the event horizon, in the domain where static NUBS exist. Alternatively, one can start with rotating MP UBS solutions and then construct the set of rotating NUBS configurations from the stationary perturbative nonuniform solutions.

The paper is structured as follows: we begin with a presentation of the general ansatz and the generic properties of rotating black strings with equal angular momenta in even spacetime dimensions. In Section 3, we consider the corresponding MP uniform solutions, discussing their thermodynamical properties and the issue of GL instability. In Section 4 we demonstrate that for $D=6$ a set of rotating NUBS solutions with two equal angular momenta exists, at least within the scope of our numerical approximation. The numerical methods used here are similar to those employed to obtain the $D=5,6$ static NUBS solutions in [5]. We discuss charged NUBS in heterotic string theory in section 5, and give our conclusions and remarks in the final section.

\section{General ansatz and properties of the solutions}

\subsection{The equations}

We consider the Einstein action

$$
I=\frac{1}{16 \pi G_{D}} \int_{M} d^{D} x \sqrt{-g} R-\frac{1}{8 \pi G_{D}} \int_{\partial M} d^{D-1} x \sqrt{-h} K
$$

in a $D$-dimensional spacetime, with $D \geq 6$ an even number. The last term in (2.1) is

the Hawking-Gibbons surface term [22], which is required in order to have a well-defined 
variational principle. $K$ is the trace of the extrinsic curvature for the boundary $\partial \mathcal{M}$ and $h$ is the induced metric of the boundary.

We consider black string solutions approaching asymptotically the $(D-1)$-dimensional Minkowski-space times a circle $\mathcal{M}^{D-1} \times S^{1}$. We denote the compact direction as $z=x^{D-1}$ and the directions of $R^{D-2}$ as $x^{1}, \ldots, x^{D-2}$, while $x^{D}=t$. The direction $z$ is periodic with period $L$. We also define the radial coordinate $r$ by $r^{2}=\left(x^{1}\right)^{2}+\cdots+\left(x^{D-2}\right)^{2}$.

To obtain nonuniform generalizations of the rotating uniform black string MP solutions, we consider space-times with $(D-2) / 2$ commuting Killing vectors $\partial_{\varphi_{k}}$. While the general configuration will then possess $(D-2) / 2$ independent angular momenta, we here restrict to rotating NUBS whose angular momenta have all equal magnitude. Analogous to the case of black holes [17, the metric parametrization then simplifies considerably for such rotating NUBS

$$
\begin{aligned}
& d s^{2}=-e^{2 A(r, z)} \frac{f(r)}{h(r)} d t^{2}+e^{2 B(r, z)}\left(\frac{d r^{2}}{f(r)}+d z^{2}\right)+e^{2 C(r, z)} r^{2} \sum_{i=1}^{(D-4) / 2}\left(\prod_{j=0}^{i-1} \cos ^{2} \theta_{j}\right) d \theta_{i}^{2} \\
& +e^{2 G(r, z)} h(r) r^{2} \sum_{k=1}^{(D-2) / 2}\left(\prod_{l=0}^{k-1} \cos ^{2} \theta_{l}\right) \sin ^{2} \theta_{k}\left(d \varphi_{k}-W(r, z) d t\right)^{2}+r^{2}\left(e^{2 C(r, z)}-e^{2 G(r, z)} h(r)\right) \\
& \times\left\{\sum_{k=1}^{(D-2) / 2}\left(\prod_{l=0}^{k-1} \cos ^{2} \theta_{l}\right) \sin ^{2} \theta_{k} d \varphi_{k}^{2}-\left[\sum_{k=1}^{(D-2) / 2}\left(\prod_{l=0}^{k-1} \cos ^{2} \theta_{l}\right) \sin ^{2} \theta_{k} d \varphi_{k}\right]\right\},
\end{aligned}
$$

where $\theta_{i} \in[0, \pi / 2]$ for $i=1, \ldots,(D-4) / 2, \varphi_{k} \in[0,2 \pi]$ for $k=1, \ldots,(D-2) / 2$, and we formally define $\theta_{0} \equiv 0, \theta_{(D-2) / 2} \equiv \pi / 2$. As a result of taking all angular momenta to be equal, the symmetry group of this spacetime is enhanced from $R \times U(1)^{(D-2) / 2}$ to $R \times U\left(\frac{D-2}{2}\right)$, where $R$ denotes time translation.

We shall assume that the information on the NUBS solutions is encoded in the functions $A(r, z), B(r, z), C(r, z), G(r, z)$ and $W(r, z)$, while $f(r)$ and $h(r)$ are two 'background' functions which are chosen for convenience. A useful parametrization when studying unstable modes around a MP solution is

$$
f(r)=1-\frac{2 M}{r^{D-4}}+\frac{2 M a^{2}}{r^{D-2}}, \quad h(r)=1+\frac{2 M a^{2}}{r^{D-2}}, \quad w(r)=\frac{2 M a}{r^{D-2} h(r)},
$$

where $M$ and $a$ are two constants related to the solution's mass and angular momentum (and $W(r, z)=w(r)$ for uniform black strings). When constructing nonperturbative NUBS solutions, a more convenient choice for the numerics is

$$
f(r)=1-\left(r_{0} / r\right)^{D-4}, \quad h(r)=1
$$

together with a redefinition of the radial coordinate, where $r_{0}$ denotes the coordinate value of the horizon. The static NUBS ansatz used in previous studies is recovered for $G(r, z)=C(r, z)$ and $W(r, z)=0$. 
A suitable combination of the Einstein equations, $G_{t}^{t}=0, G_{r}^{r}+G_{z}^{z}=0, G_{\theta_{1}}^{\theta_{1}}=0, G_{\varphi_{1}}^{t}=0$ and $G_{\varphi_{1}}^{\varphi_{1}}=0$, yields the following set of equations for the functions $A, B, C, G$ and $W$

$$
\begin{aligned}
& \hat{O}^{2} A+(\hat{O} A)^{2}+\hat{O} A \cdot \hat{O} G+(D-4) \hat{O} A \cdot \hat{O} C-\frac{e^{-2 A+2 G} h^{2}}{2 r^{2} f}(\hat{O} W)^{2} \\
& +\left(\frac{D-3}{r}+\frac{3 f^{\prime}}{2 f}-\frac{h^{\prime}}{2 h}\right) \partial_{r} A+\left(\frac{f^{\prime}}{2 f}-\frac{h^{\prime}}{2 h}\right)\left((D-4) \partial_{r} C+\partial_{r} G\right) \\
& +\frac{(D-3) f^{\prime}}{2 r f}-\frac{(D-3) h^{\prime}}{2 r h}-\frac{f^{\prime} h^{\prime}}{2 f h}+\frac{h^{\prime 2}}{2 h^{2}}+\frac{f^{\prime \prime}}{2 f}-\frac{h^{\prime \prime}}{2 h}=0, \\
& \hat{O}^{2} B-\frac{e^{-2 A+2 G} r^{2} h^{2}}{4 f}(\hat{O} W)^{2}-(D-4) \hat{O} A \cdot \hat{O} C-\frac{1}{2}(D-4)(D-5)(\hat{O} C)^{2} \\
& -\hat{O} A \cdot \hat{O} G-(D-4) \hat{O} C \cdot \hat{O} G-\left(\frac{D-3}{r}+\frac{h^{\prime}}{2 h}\right) \partial_{r} A+\frac{f^{\prime}}{2 f} \partial_{r} B-(D-4)\left(\frac{D-4}{r}+\frac{f^{\prime}}{2 f}\right) \partial_{r} C \\
& +\frac{1}{2}\left(\frac{h^{\prime}}{h}-\frac{f^{\prime}}{f}-\frac{2(D-4)}{r}\right) \partial_{r} G+\frac{(D-2)(D-4)}{2 r^{2} f} e^{2 B-2 C}-\frac{(D-4) h}{2 r^{2} f} e^{2 B-4 C+2 G} \\
& -\frac{(D-3) f^{\prime}}{2 r f}-\frac{f^{\prime} h^{\prime}}{4 f h}+\frac{h^{\prime}}{2 r h}+\frac{h^{\prime 2}}{4 h^{2}}-\frac{(D-3)(D-4)}{2 r^{2}}=0, \\
& \hat{O}^{2} C+(D-4)(\hat{O} C)^{2}+\hat{O} C \cdot \hat{O} G+\hat{O} A \cdot \hat{O} C+\frac{1}{r}\left(\partial_{r} A+\partial_{r} G\right) \\
& +\left(\frac{2 D-7}{r}+\frac{f^{\prime}}{f}\right) \partial_{r} C-\frac{(D-2)}{r^{2} f} e^{2 B-2 C}+\frac{2 h}{r^{2} f} e^{2 B-4 C+2 G}+\frac{f^{\prime}}{r f}+\frac{D-4}{r^{2}}=0, \\
& \hat{O}^{2} G+(\hat{O} G)^{2}+\hat{O} A \cdot \hat{O} G+(D-4) \hat{O} C \cdot \hat{O} G+\frac{e^{-2 A+2 G} r^{2} h^{2}}{2 f}(\hat{O} W)^{2} \\
& +\frac{(D-4)}{2}\left(\frac{2}{r}+\frac{h^{\prime}}{h}\right) \partial_{r} C+\left(\frac{f^{\prime}}{f}+\frac{h^{\prime}}{2 h}+\frac{(D-2)}{r}\right) \partial_{r} G+\left(\frac{1}{r}+\frac{h^{\prime}}{2 h}\right) \partial_{r} A-\frac{(D-4) h}{r^{2} f} e^{2 B-4 C+2 G} \\
& +\frac{f^{\prime}}{r f}+\left(\frac{D-3}{2 r}+\frac{f^{\prime}}{2 f}-\frac{h^{\prime}}{2 h}\right) \frac{h^{\prime}}{h}+\frac{h^{\prime \prime}}{2 h}+\frac{D-4}{r^{2}}=0 \\
& \hat{O}^{2} W-\hat{O} A \cdot \hat{O} W+(D-4) \hat{O} C \cdot \hat{O} W+3 \hat{O} G \cdot \hat{O} W+\left(\frac{D-1}{r}+\frac{2 h^{\prime}}{h}\right) \partial_{r} W=0,
\end{aligned}
$$

where we define

$$
\hat{O} U \cdot \hat{O} V=\partial_{r} U \partial_{r} V+\frac{1}{f} \partial_{z} U \partial_{z} V, \quad \hat{O}^{2} U=\partial_{r}^{2} U+\frac{1}{f} \partial_{z}^{2} U
$$

and a prime denotes the derivative with respect to $r$.

All other Einstein equations except for $G_{z}^{r}=0$ and $G_{r}^{r}-G_{z}^{z}=0$ are linear combinations of those used to derive the above equations or are identically zero. The remaining Einstein 
equations $G_{z}^{r}=0, G_{r}^{r}-G_{z}^{z}=0$ yield two constraints. Following [3], we note that setting $G_{t}^{t}=G_{\theta_{i}}^{\theta_{i}}=G_{\varphi_{k}}^{\varphi_{k}}=G_{r}^{r}+G_{z}^{z}=0$ in $\nabla_{\mu} G^{\mu r}=0$ and $\nabla_{\mu} G^{\mu z}=0$, we obtain

$$
\begin{gathered}
\partial_{z}\left(\sqrt{-g} G_{z}^{r}\right)+\sqrt{f} \partial_{r}\left(\sqrt{f} \sqrt{-g} \frac{1}{2}\left(G_{r}^{r}-G_{z}^{z}\right)\right)=0 \\
\sqrt{f} \partial_{r}\left(\sqrt{-g} G_{z}^{r}\right)-\partial_{z}\left(\sqrt{f} \sqrt{-g} \frac{1}{2}\left(G_{r}^{r}-G_{z}^{z}\right)\right)=0
\end{gathered}
$$

and, defining $\hat{r}$ via $\partial / \partial_{\hat{r}}=\sqrt{f} \partial / \partial_{r}$, then yields the Cauchy-Riemann relations

$$
\begin{aligned}
& \partial_{z}\left(\sqrt{-g} G_{z}^{r}\right)+\partial_{\hat{r}}\left(\sqrt{f} \sqrt{-g} \frac{1}{2}\left(G_{r}^{r}-G_{z}^{z}\right)\right)=0, \\
& \partial_{\hat{r}}\left(\sqrt{-g} G_{z}^{r}\right)-\partial_{z}\left(\sqrt{f} \sqrt{-g} \frac{1}{2}\left(G_{r}^{r}-G_{z}^{z}\right)\right)=0 .
\end{aligned}
$$

Thus the weighted constraints satisfy Laplace equations, and the constraints are fulfilled, when one of them is satisfied on the boundary and the other at a single point [3].

\subsection{General properties}

We impose the event horizon to reside at a surface of constant radial coordinate $r=r_{0}$, where $f(r)=f^{\prime}\left(r_{0}\right)\left(r-r_{0}\right)+O\left(r-r_{0}\right)^{2}$ while $h\left(r_{0}\right)>0, f^{\prime}\left(r_{0}\right)>0$. Also, the functions $f(r)$ and $h(r)$ take only positive values for $r>r_{0}$ and tend to one for $r \rightarrow \infty$.

The Killing vector $\chi=\partial / \partial_{t}+\sum_{k} \Omega_{k} \partial / \partial \varphi_{k}$ is orthogonal to and null on the horizon. For the solutions within the ansatz (2.2), the event horizon angular velocities are equal, $\Omega_{k}=\Omega_{H}=\left.W(r, z)\right|_{r=r_{0}}$.

Utilizing the reflection symmetry of the nonuniform black strings w.r.t. $z=L / 2$, the nonuniform solutions are constructed subject to the following set of boundary conditions

$$
\begin{gathered}
\left.A\right|_{r=\infty}=\left.B\right|_{r=\infty}=\left.C\right|_{r=\infty}=\left.G\right|_{r=\infty}=\left.W\right|_{r=\infty}=0, \\
\left.B\right|_{r=r_{0}}-\left.A\right|_{r=r_{0}}=d_{0},\left.\partial_{r} A\right|_{r=r_{0}}=\left.\partial_{r} C\right|_{r=r_{0}}=\left.\partial_{r} G\right|_{r=r_{0}}=0,\left.\quad W\right|_{r=r_{0}}=\Omega_{H}, \\
\left.\partial_{z} A\right|_{z=0, L / 2}=\left.\partial_{z} B\right|_{z=0, L / 2}=\left.\partial_{z} C\right|_{z=0, L / 2}=\left.\partial_{z} G\right|_{z=0, L / 2}=\left.\partial_{z} W\right|_{z=0, L / 2}=0,
\end{gathered}
$$

where the constant $d_{0}$ is related to the Hawking temperature of the solutions.

As in the case of $3+1$-dimensional Kerr black holes, the rotating black strings have an ergosurface inside of which observers cannot remain stationary, and will move in the direction of the rotation. The ergosurface is located at $g_{t t}=0$, i.e.

$$
e^{2 G(r, z)} r^{2} h(r) W(r, z)^{2}-\frac{e^{2 A(r, z)} f(r)}{h(r)}=0
$$

and does not intersect the horizon. (Note that the ergoregion here extends nontrivially in the extra dimension.) 
The computation of the conserved charges of rotating black strings was discussed e.g. in [23]. The essential idea there is to consider the asymptotic values of the gravitational field far away from the black string and to compare them with those corresponding to a gravitational field in the absence of the black string. The obvious choice of the background in this case is $\mathcal{M}^{D-1} \times S^{1}$, the asymptotic form of the relevant metric components being

$$
g_{t t} \simeq-1+\frac{c_{t}}{r^{D-4}}, \quad g_{z z} \simeq 1+\frac{c_{z}}{r^{D-4}}, \quad g_{\varphi_{k} t} \simeq\left(\prod_{l=0}^{k-1} \cos ^{2} \theta_{l}\right) \sin ^{2} \theta_{k} \frac{c_{\varphi}}{r^{D-4}},
$$

which reveals the existence of three free parameters $c_{t}, c_{z}$ and $c_{\varphi}$. The mass-energy $E$, the tension $\mathcal{T}$ and the angular momenta $J_{k}$ of black string solutions are given by

$$
E=\frac{A_{D-3} L}{16 \pi G_{D}}\left((D-3) c_{t}-c_{z}\right), \mathcal{T}=\frac{A_{D-3}}{16 \pi G_{D}}\left(c_{t}-(D-3) c_{z}\right), J_{k}=J=-\frac{A_{D-3} L}{8 \pi G_{D}} c_{\varphi},
$$

where $A_{D-3}=2 \pi^{\frac{D-2}{2}} / \Gamma((D-2) / 2)$ is the area of the unit $D-3$ sphere.

The global charges of a black string can also be computed by using the quasilocal tensor of Brown and York [24], augmented by the counterterms formalism. In this approach we add to the action (2.1) suitable counterterms $I_{c t}$ built up with curvature invariants of the induced metric on the boundary $\partial \mathcal{M}$ [25], 226], 27]. These counterterms do not alter the bulk equations of motion. Unlike the background substraction, this procedure is satisfying since it is intrinsic to the spacetime of interest and it is unambiguous once the counterterm is specified. Our choice of the counterterm was similar to the static case [5], $I_{c t}=-\frac{1}{8 \pi G_{D}} \sqrt{(D-3) /(D-4)} \int_{\partial \mathcal{M}} d^{D-1} x \sqrt{-h} \sqrt{\mathcal{R}}$, where $\mathcal{R}$ is the Ricci scalar of the boundary geometry. The variation of the total action with respect to the boundary metric $h_{i j}$ provides a boundary stress-tensor, whose expression is given e.g. in [5]. The mass-energy, tension and angular momenta are the charges associated to $\partial / \partial t, \partial / \partial z$ and $\partial / \partial \varphi_{k}$ respectively (note that $\partial / \partial z$ is a Killing symmetry of the boundary metric). We have verified that for $D=6,8$ and $D=10$ the expressions computed in this way agree with (2.18) (see 26, 28] for similar computations for a different type of rotating solutions).

One can also define a relative tension $n$ (also called the relative binding energy or scalar charge)

$$
n=\frac{\mathcal{T} L}{E}=\frac{c_{t}-(D-3) c_{z}}{(D-3) c_{t}-c_{z}}
$$

which measures how large the tension is relative to the mass-energy, being constant for UBS solutions.

The Hawking temperature $T_{H}=\kappa_{H} / 2 \pi$ can be obtained from the standard relation

$$
\kappa_{H}^{2}=-\left.\frac{1}{2} \nabla^{a} \chi^{b} \nabla_{a} \chi_{b}\right|_{r=r_{0}}
$$

where $\kappa_{H}$ is the surface gravity, which is constant at the horizon. One finds

$$
T_{H}=e^{A_{0}-B_{0}} T_{H}^{(0)}=e^{-d_{0}} T_{H}^{(0)},
$$


where $T_{H}^{(0)}$ is the Hawking temperature of the uniform solution based on the same 'background' functions (2.3), $T_{H}^{(0)}=f^{\prime}\left(r_{0}\right) /\left(4 \pi \sqrt{h\left(r_{0}\right)}\right)$. Here and below $A_{0}(z), B_{0}(z), C_{0}(z), G_{0}(z)$ and $W_{0}(z)$ denote the values of the metric functions on the event horizon $r=r_{0}$.

The area $A_{H}$ of the black string horizon can also be expressed in a similar way

$$
A_{H}=A_{H}^{(0)} \frac{1}{L} \int_{0}^{L} e^{B_{0}+(D-4) C_{0}+G_{0}} d z
$$

with $A_{H}^{(0)}$ the event horizon area of the corresponding uniform solution

$$
A_{H}^{(0)}=L A_{D-3} r_{0}^{D-3} \sqrt{h\left(r_{0}\right)}
$$

As usual, one identifies the entropy of the black string solutions with one quarter of their event horizon area, $S=A_{H} / 4 G_{D}$.

Considering the thermodynamics of these solutions, the black strings should satisfy the first law of thermodynamics

$$
d E=T_{H} d S+\frac{(D-2)}{2} \Omega_{H} d J+\mathcal{T} d L
$$

One may regards the parameters $S, J$ and $L$ as a complete set of extensive parameters for the mass-energy $E(S, J, L)$ and define the intensive parameters conjugate to them. These quantities are the temperature, the angular velocities and the tension.

Following [13], 29], one can derive in a simple way a Smarr formula, by letting the length of the compact extra dimension change as $L \rightarrow L+d L$. This implies

$$
E \rightarrow E\left(1+\frac{d L}{L}\right)^{D-3}, \quad S \rightarrow S\left(1+\frac{d L}{L}\right)^{D-2}, \quad J \rightarrow J\left(1+\frac{d L}{L}\right)^{D-2} .
$$

As a result we find the Smarr formula (see also [23] for a different derivation of this relation)

$$
\frac{D-3-n}{D-2} E=T_{H} S+\frac{(D-2)}{2} \Omega_{H} J
$$

In the canonical ensemble, we study black strings holding the temperature $T_{H}$, the angular momenta $J$ and the length $L$ of the extra dimension fixed. The associated thermodynamic potential is the Helmholz free energy

$$
F\left[T_{H}, J, L\right]=E-T_{H} S
$$

In the grand canonical ensemble, on the other hand, we keep the temperature, the angular velocity and the tension fixed. In this case the thermodynamics is obtained from the Gibbs potential

$$
G\left[T_{H}, \Omega_{H}, \mathcal{T}\right]=E-T_{H} S-\frac{(D-2)}{2} \Omega_{H} J-\mathcal{T} L
$$


We finally remark that the technique used in [30], [31] to construct 'copies of solutions' works for rotating NUBS, too. When taking $f(r)=1-\left(r_{0} / r\right)^{D-4}, h(r)=1$, i.e. (2.4), the Einstein equations (2.5)-(2.9) are left invariant by the transformation $r \rightarrow r / k, z \rightarrow z / k$, $r_{0} \rightarrow r_{0} / k$, with $k$ an arbitrary positive integer. Therefore, one may generate a family of vacuum solutions in this way. The new solutions have the same length of the extra dimension. Their relevant properties, expressed in terms of the corresponding properties of the initial solution, read

$$
E^{(k)}=\frac{E}{k^{D-4}}, T_{H}^{(k)}=k T_{H}, \quad S^{(k)}=\frac{S}{k^{D-3}}, n^{(k)}=n, J^{(k)}=\frac{J}{k^{D-3}}, \Omega_{H}^{(k)}=k \Omega_{H} .
$$

\section{Rotating UBS with equal angular momenta}

\subsection{Thermodynamics}

We start by discussing the properties of uniform black string solutions obtained by taking $A=B=C=G=0$ in the general ansatz (2.2) with the functions $f, h$ and $W(r, z)=w(r)$ given by (2.3). The extra dimension plays no role here and the general results apply to $d=(D-1)$-dimensional MP black holes when formally taking $L=1$ in the relations below.

The uniform black strings have two parameters $M$ and $a$ which, from (2.18), are related to the physical mass-energy and angular momenta by

$$
E=\frac{A_{D-3} L}{8 \pi G_{D}}(D-3) M, \quad J_{i}=J=\frac{A_{D-3} L}{4 \pi G_{D}} M a,
$$

Hence one can think of $a$ as essentially the angular momentum per unit mass. The tension $\mathcal{T}$ of the uniform solutions is fixed by the mass-energy $E$ and length $L$ of the extra dimension

$$
\mathcal{T}=\frac{E}{L(D-3)}
$$

The event horizon of these uniform black strings can be determined as the largest root of $1 / g_{r r}=0$ resp. $f(r)=0$. That is

$$
r_{0}^{D-2}-2 M r_{0}^{2}+2 M a^{2}=0 .
$$

This equation has zero, one or two positive roots, depending on the sign of $f\left(r_{s}\right)$, where $r_{s}=(4 M /(D-2))^{1 /(D-4)}$ is the largest root of $\left(r^{D-2} f(r)\right)^{\prime}=0$. The existence of a regular horizon implies an upper limit on $a$,

$$
a^{2} \leq \frac{D-4}{D-2}\left(\frac{4 M}{D-2}\right)^{\frac{2}{D-4}}=a_{\max }^{2}
$$

which via (3.1) can also be expressed as

$$
\frac{J}{E^{\frac{D-3}{D-4}}} \leq \sqrt{D-4}\left(\frac{2^{D+1} \pi}{(D-3)^{D-3}(D-2)^{(D-2) / 2}}\right)^{1 /(D-4)}\left(\frac{G_{D}}{A_{D-3} L}\right)^{1 /(D-4)} .
$$


This strongly contrasts with the case of MP UBS solutions with a single nonzero angular momentum, where for $D>6$ there are configurations with arbitrarily large $J$, without an occurrence of an extremal limit ${ }^{1}$.

The ergosurface of the uniform solutions is located at $r_{e}=(2 M)^{1 /(D-4)}$. The horizon angular velocities and the Hawking temperature of the UBS solutions are given by

$$
\Omega_{H}=\frac{a}{r_{0}^{2}}, \quad T_{H}=\frac{1}{4 \pi r_{0}}\left(\frac{2 M}{r_{0}^{D-4}}\right)^{1 / 2}\left((D-2) \frac{r_{0}^{D-4}}{2 M}-2\right) .
$$

Solutions with $a=a_{\max }$ are extremal black strings with $T_{H}=0$, possessing a nonzero entropy. As can be seen e.g. by computing the Kretschmann scalar, the hypersurface $r=r_{0}$ is not singular in this limit.

Similar to the static case, the gravitational thermodynamics of the rotating UBS can be formulated via the Euclidean path integral. The Euclidean spinning black string solutions can be obtained from the Minkowskian ones by sending $t \rightarrow-i t$ and $a \rightarrow i a$ (complexifying $a$ is necessary in order to keep the $d t d \varphi_{i}$ part of the metric real). The thermodynamic system has a constant temperature $T_{H}=1 / \beta$ which is determined by requiring the Euclidean section be free of conical singularities (the temperature computed in this way coincides with that in (3.6)). The partition function for the gravitational field is defined by a sum over all smooth Euclidean geometries which are periodic with a period $\beta$ in imaginary time [22]. This integral is computed by using the saddle point approximation, the global charges and entropy of the solutions being evaluated by standard thermodynamic formulae. Upon application of the Gibbs-Duhem relation to the partition function [33], this yields an expression for the entropy

$$
S=\beta\left(E-\frac{(D-2)}{2} \Omega_{H} J-\mathcal{T} L\right)-I,
$$

(with $I$ the regularized tree level Euclidean action), which agrees with that computed from (2.22). The entropy can be written in terms of $M, r_{0}$ as

$$
S=\frac{1}{4 G_{D}} A_{D-3} L r_{0}^{D-3}\left(\frac{2 M}{r_{0}^{D-4}}\right)^{1 / 2} .
$$

The parameters $M, a, r_{0}$ can be eliminated and one can write the following equation of state (analogous to $f(p, V, T)$, for, say, a gas at pressure $p$ and volume $V$ )

$$
\begin{aligned}
J=2^{-(D+6) / 2} \frac{A_{D-3} L}{G_{D}}(D-4)^{D-2} \pi^{1-D} \Omega_{H} T_{H}^{2-D}\left(1+\sqrt{1+\frac{(D-4) \Omega_{H}^{2}}{2 \pi^{2} T_{H}^{2}}}\right)^{-D / 2} \\
\times\left((D-2) \sqrt{1+\frac{(D-4) \Omega_{H}^{2}}{2 \pi^{2} T_{H}^{2}}}-D+6\right)^{-(D-4) / 2}
\end{aligned}
$$

\footnotetext{
${ }^{1}$ However, as argued in [32], MP black holes with a single nonzero angular momentum are in fact classically unstable (at least for large rotation) and an effective Kerr bound arises through a dynamical decay mechanism.
} 
The following relations are also useful in what follows

$$
\begin{aligned}
& T_{H}=\frac{D-4}{\pi}\left(\frac{A_{D-3} L}{2^{2(D-2)} G_{D}}\right)^{\frac{1}{D-3}} S^{1 /(3-D)}\left(1+\frac{4 \pi^{2} J^{2}}{S^{2}}\right)^{-\frac{D-4}{2(D-3)}}\left(1-\frac{8 \pi^{2} J^{2}}{(D-4) S^{2}}\right) \\
& E=2^{-\frac{D+6}{2}}(D-3)(D-4)^{D-4} \pi^{3-D} \frac{A_{D-3} L}{G_{D}} T_{H}^{4-D}\left(1+\sqrt{1+\frac{(D-4) \Omega_{H}^{2}}{2 \pi^{2} T_{H}^{2}}}\right)^{(2-D) / 2} \\
& \times\left((D-2) \sqrt{1+\frac{(D-4) \Omega_{H}^{2}}{2 \pi^{2} T_{H}^{2}}}+6-D\right)^{(6-D) / 2}, \\
& S=2^{-(D+2) / 2} \frac{A_{D-3} L}{G_{D}}(D-4)^{D-3} \pi^{3-D} T_{H}^{3-D}\left(1+\sqrt{1+\frac{(D-4) \Omega_{H}^{2}}{2 \pi^{2} T_{H}^{2}}}\right)^{-(D-2) / 2} \\
& \times\left((D-2) \sqrt{1+\frac{(D-4) \Omega_{H}^{2}}{2 \pi^{2} T_{H}^{2}}}-D+6\right)^{-(D-4) / 2}, \\
& E=2^{-\frac{2(D-2)}{(D-3)}} \frac{(D-3)}{\pi}\left(\frac{A_{D-3} L}{G_{D}}\right)^{1 /(D-3)} S^{\frac{D-4}{D-3}}\left(1+\frac{4 \pi^{2} J^{2}}{S^{2}}\right)^{\frac{D-2}{2(D-3)}} .
\end{aligned}
$$

As implied by (3.10), the rotating UBSs have always a smaller temperature for the same entropy than the static UBSs. The energy to entropy ratio of the UBS solutions satisfies the following bounds

$$
\frac{1}{2^{2(D-2)}} \leq\left(\frac{\pi}{D-3}\right)^{D-3} \frac{G_{D}}{A_{D-3} L} \frac{E^{D-3}}{S^{D-4}} \leq \frac{(D-2)^{(D-2) / 2}}{2^{5(D-2) / 2}},
$$

these limits being approached for static and extremal solutions, respectively. There is also an upper bound for the ratio $J / S$,

$$
\frac{J}{S} \leq \frac{1}{2 \pi} \sqrt{\frac{D-4}{2}}
$$

The analysis of the thermodynamic stability of the rotating UBS solutions can be performed using the above relations. It is known that different thermodynamic ensembles are not exactly equivalent and may not lead to the same conclusions as they correspond to different physical situations. Mathematically, thermodynamic stability is equated with the subadditivity of the entropy function. This requires $S(E, J, L)$ to be a concave function of its extensive variables. The stability can also be studied by the behaviour of the energy $E(S, J, L)$ which should be a convex function. Therefore one has to compute the determinant of the Hessian matrix of $E(S, J, L)$ with respect to its extensive variables $X_{i}, H_{X_{i} X_{j}}^{E}=\left[\partial^{2} E / \partial X_{i} \partial X_{j}\right][34$, [35]. 
In the canonical ensemble, the subadditivity of the entropy is exactly equivalent to positivity of the specific heat at constant $(J, L), C_{J, L}=T_{H}\left(\partial S / \partial T_{H}\right)_{J, L}$. Also, the Gibbs potential which is relevant for a grand canonical ensemble can be written as $G\left[T_{H}, \Omega_{H}, L\right]=E /(D-2)$, as a result of the Smarr law (2.26).

At this point it is instructive to see first the corresponding situation for the $(D-1)$ dimensional MP black holes. It is easy to work out from (3.10) that the condition for a positive specific heat at fixed angular momenta is equivalent to

$$
32(D-1) \pi^{4} J^{4}+4(D(D-5)+10) \pi^{2} J^{2} S^{2}-(D-4) S^{4}>0 .
$$

As expected, $C_{J}$ is negative far from extremality, becoming positive for large enough values of $J$.

To discuss the thermodynamic stability of black holes in a grand canonical ensemble, we consider first the specific heat at constant angular velocity at the horizon

$$
C_{\Omega}=T_{H}\left(\frac{\partial S}{\partial T_{H}}\right)_{\Omega_{H}}
$$

A straightforward computation using (3.12) shows that this is a negative quantity in the full range of variables, $C_{\Omega}<0$. One can also verify that the determinant of the Hessian matrix of $E(S, J)$ is negative

$$
\operatorname{det}\left(\partial^{2} E / \partial X_{i} \partial X_{j}\right)=-\frac{4(D-2) \pi^{2}}{(D-3)^{3}} \frac{E^{2}\left(8 J^{2} \pi^{2}+(D-2) S^{2}\right)}{S^{2}\left(4 J^{2} \pi^{2}+S^{2}\right)}<0 .
$$

As a result, all MP rotating black hole solutions with equal angular momenta are unstable in a grand canonical ensemble, and also the configurations far from extremality in a canonical ensemble.

Another 'response function' of interest is the 'isothermal permittivity'

$$
\epsilon_{T} \equiv\left(\frac{\partial J}{\partial \Omega_{H}}\right)_{T_{H}} .
$$

One finds from (3.9) that the condition for a positive $\epsilon_{T}$ is

$$
\frac{\Omega_{H}}{T_{H}}<\left(\frac{\pi^{2}}{D-4}\left(\frac{2+5 D-D^{2}}{(D-2)(D-3)}+\sqrt{\frac{D^{2}-5 D+22}{(D-2)(D-3)}}\right)\right)^{1 / 2} .
$$

For $D=6$, this corresponds to $\Omega_{H} / T_{H}<1.7165$, for $D=8$ to $\Omega_{H} / T_{H}<0.7892$ and for $D=10$ to $\Omega_{H} / T_{H}<0.477$. All other rotating black hole solutions have a negative 'isothermal permittivity' and thus are unstable to angular fluctuations, both in a grand canonical and a canonical ensemble.

These conclusions remain unchanged when adding one (trivial) extra dimension to the black hole solutions. Similar to the static case, all grand canonical configurations are thermally 
unstable $^{2}$. Also, all rotating UBS solutions with $J / S<J^{(c)} / S^{(c)}$ are unstable in a canonical ensemble, where the critical ratio $J^{(c)} / S^{(c)}$ is given by

$$
\frac{J^{(c)}}{S^{(c)}}=\frac{1}{4 \pi \sqrt{D-1}}(\sqrt{(D-2)(D-3)(D(D-5)+22)}-D(D-5)-10)^{1 / 2} .
$$

At the critical point, the specific heat goes through an infinite discontinuity, and a second order phase transition takes place. The critical values for other relevant quantities read

$$
T_{H}^{(c)}=f_{1}(D)\left(J^{(c)}\right)^{-\frac{1}{D-3}}, \quad E^{(c)}=f_{2}(D)\left(J^{(c)}\right)^{\frac{D-4}{D-3}}, \quad \Omega_{H}^{(c)}=f_{3}(D)\left(J^{(c)}\right)^{-\frac{1}{D-3}},
$$

where

$$
\begin{aligned}
f_{1}(D)= & 2^{-\frac{2 D-1}{D-3}}\left(\frac{A_{D-3} L}{G_{D}}\right)^{1 /(D-3)} \pi^{-\frac{D-2}{D-3}}(D-1)^{-\frac{(D-1)}{2(D-3)}}\left(f_{0}(D)-(D-2)(D-7)\right)^{-\frac{D-4}{2(D-3)}} \\
& \times\left(3(D-2)(D-3)-f_{0}(D)\right)\left(f_{0}(D)-18+5 D-D^{2}\right)^{\frac{1}{2(D-3)}} \\
f_{2}(D)= & 2^{-\frac{D+2}{D-3}}\left(\frac{A_{D-3} L}{G_{D}}\right)^{1 /(D-3)} \pi^{1 /(3-D)}(D-3)(D-1)^{1 /(3-D)} \\
& \times\left(f_{0}(D)-(D-2)(D-7)\right)^{\frac{D-2}{2(D-3)}}\left(f_{0}(D)-D(D-5)-10\right)^{-\frac{D-4}{2(D-3)}} \\
f_{3}(D)= & (32 \pi)^{1 /(3-D)}\left(\frac{A_{D-3} L}{G_{D}}\right)^{1 /(D-3)}(D-1)^{1 /(3-D)}\left(f_{0}-(D-2)(D-7)\right)^{-\frac{D-4}{2(D-3)}} \\
& \times\left(f_{0}(D)-D(D-5)-10\right)^{\frac{D-2}{2(D-3)}}
\end{aligned}
$$

while

$$
f_{0}(D)=\sqrt{(D-2)(D-3)(D(D-5)+22)} .
$$

One finds for example the critical ratio $\Omega_{H}^{(c)} / T_{H}^{(c)} \simeq 2.42757$ for $D=6, \Omega_{H}^{(c)} / T_{H}^{(c)} \simeq 1.1162$ for $D=8$ and $\Omega_{H}^{(c)} / T_{H}^{(c)} \simeq 0.6747$ for $D=10$.

\subsection{Gregory-Laflamme instability}

It is natural to expect that the MP uniform black strings become unstable at critical values

of the mass and angular momentum. To determine these critical values for black strings with equal magnitude angular momenta, we make an expansion around the UBS of the form

$$
\begin{aligned}
& A(r, z)=\epsilon a_{1}(r) \cos (k z)+O\left(\epsilon^{2}\right), \\
& B(r, z)=\epsilon b_{1}(r) \cos (k z)+O\left(\epsilon^{2}\right), \\
& C(r, z)=\epsilon c_{1}(r) \cos (k z)+O\left(\epsilon^{2}\right), \\
& G(r, z)=\epsilon g_{1}(r) \cos (k z)+O\left(\epsilon^{2}\right), \\
& W(r, z)=w(r)+\epsilon w_{1}(r) \cos (k z)+O\left(\epsilon^{2}\right),
\end{aligned}
$$

with $\epsilon$ a small parameter and $f, h, w$ given by (2.3). This expansion is appropriate for

\footnotetext{
${ }^{2}$ However, in this case the determinant of the Hessian vanishes identically, as a result of the special dependence on $L$. This happens already for a Schwarzschild black string when considered as a solution in a grand canonical ensemble with fixed $T_{H}, \mathcal{T}$.
} 


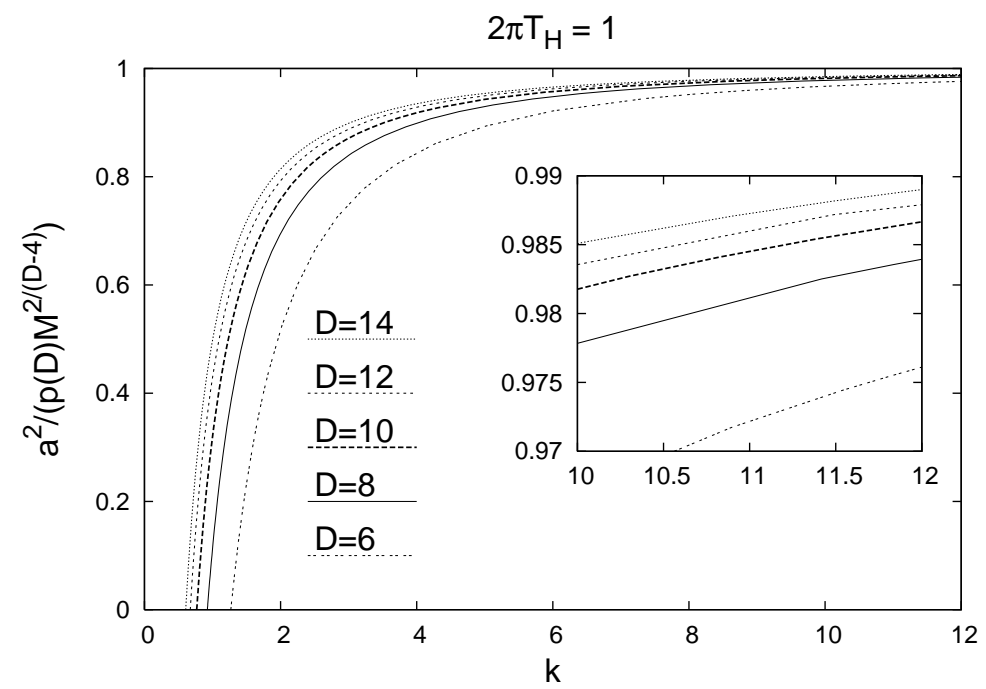

Figure 1: The dimensionless quantity $a^{2} /\left(p(D) M^{\frac{2}{D-4}}\right)$ is shown as a measure of the rotation of the uniform black strings at constant temperature $2 \pi T_{H}=1$ in $D$ even dimensions, $6 \leq D \leq 14$, versus the wavenumber $k$ of the zeromode fluctuation.

studying perturbations at the wavelength which is marginally stable.

Upon substituting the above expressions into the Einstein equations, to order $O(\epsilon)$ the following set of ODE is generated

$$
\begin{gathered}
a_{1}^{\prime \prime}+\left(\frac{D-3}{r}+\frac{3 f^{\prime}}{2 f}-\frac{h^{\prime}}{2 h}\right) a_{1}^{\prime}+\left(\frac{f^{\prime}}{2 f}-\frac{h^{\prime}}{2 h}\right)\left((D-4) c_{1}^{\prime}+g_{1}^{\prime}\right)-\frac{r^{2} h^{2} w^{\prime}}{f} w_{1}^{\prime}+\frac{r^{2} h^{2} w^{\prime 2}}{f}\left(a_{1}-g_{1}\right)-\frac{k^{2} a_{1}}{f}=0, \\
b_{1}^{\prime \prime}-\left(\frac{D-3}{r}+\frac{h^{\prime}}{2 h}\right) a_{1}^{\prime}-\frac{(D-4)^{2}}{r} c_{1}^{\prime}+\frac{f^{\prime}}{2 f}\left(b_{1}^{\prime}-(D-4) c_{1}^{\prime}\right)+\frac{1}{2}\left(\frac{h^{\prime}}{h}-\frac{f^{\prime}}{f}-\frac{2(D-4)}{r}\right) g_{1}^{\prime} \\
-\frac{r^{2} h^{2} w^{\prime}}{2 f} w_{1}^{\prime}-\frac{(D-4) h}{r^{2} f}\left(b_{1}-2 c_{1}+g_{1}\right)+\frac{r^{2} h^{2} w^{\prime 2}}{2 f}\left(a_{1}-g_{1}\right)+\frac{(D-2)(D-4)}{r^{2} f}\left(b_{1}-c_{1}\right)-\frac{k^{2} b_{1}}{f}=0, \\
c_{1}^{\prime \prime}+\frac{f^{\prime}}{f} c_{1}^{\prime}+\frac{1}{r}\left(a_{1}^{\prime}+(2 D-7) c_{1}^{\prime}+g_{1}^{\prime}\right)+\frac{2(D-2)}{r^{2} f}\left(c_{1}-b_{1}\right)+\frac{4 h}{r^{2} f}\left(b_{1}-2 c_{1}+g_{1}\right)-\frac{k^{2} c_{1}}{f}=0, \\
g_{1}^{\prime \prime}+\left(\frac{D-2}{r}+\frac{f^{\prime}}{f}+\frac{h^{\prime}}{2 h}\right) g_{1}^{\prime}+\frac{r^{2} h^{2} w^{\prime}}{f} w_{1}^{\prime}+\frac{2(D-4) h}{r^{2} f}\left(2 c_{1}-g_{1}-b_{1}\right) \\
+\left(\frac{1}{r}+\frac{h^{\prime}}{2 h}\right)\left(a_{1}^{\prime}+(D-4) c_{1}^{\prime}\right)+\frac{r^{2} h^{2} w^{2}}{f}\left(g_{1}-a_{1}\right)-\frac{k^{2} g_{1}}{f}=0, \\
w_{1}^{\prime \prime}+\left(\frac{D-1}{r}+\frac{2 h^{\prime}}{h}\right) w_{1}^{\prime}+\left(-a_{1}^{\prime}+(D-4) c_{1}^{\prime}+3 g_{1}^{\prime}\right) w^{\prime}-\frac{k^{2} w_{1}}{f}=0 .
\end{gathered}
$$

This eigenvalue problem for the wavenumber $k=2 \pi / L$ is then solved numerically with suitable boundary conditions, for rotating black strings in $D$ even dimensions, $6 \leq D \leq$ 14. The results are displayed in Figure 1, where we exhibit the dimensionless quantity (see Eqs. (3.1), (3.4)) $a^{2} /\left(p(D) M^{\frac{2}{D-4}}\right)$, with

$$
p(D)=\frac{D-4}{D-2}\left(\frac{4}{D-2}\right)^{\frac{2}{D-4}}
$$


as a measure of the rotation versus the wavenumber $k$ for constant temperature, $2 \pi T_{H}=1$. Note, that for extremal solutions $a^{2} /\left(p(D) M^{\frac{2}{D-4}}\right)=1$. Keeping the temperature fixed, the wavenumber $k$ of the marginally stable mode increases with increasing rotation and decreases with increasing dimension $D$.

Introducing the scaled energy-mass $M_{s}$ and the scaled angular momentum $J_{s}$ (following Eq. (2.18)

$$
E=\frac{A_{D-3} L}{16 \pi G_{D}}(D-3) M_{s}, J_{k}=J=-\frac{A_{D-3} L}{8 \pi G_{D}} J_{s},
$$

these results are presented in Figure 2 (left) in a "phase diagram" format for fixed $L=L_{0}$, where $L_{0}$ there corresponds to the critical length of the corresponding static solutions. Here $M_{s}$ and $J_{s}$ are suitably normalized and equipped with powers, such that the extremality curve is the same for any dimension $D$.

In principle, following [1], one can get an estimation of $k$ by equating the entropy of the rotating string with that of a MP rotating black hole with the same momenta. The entropy, mass-energy and angular momenta of a black string are related through (3.13). The corresponding relation for a MP black hole in $D$ dimensions with $(D-2) / 2$ equal angular momenta can easily be derived by using the relations in [15], and reads

$$
E=\frac{(D-2)}{\pi} 2^{-\frac{2(D-1)}{D-2}}\left(\frac{A_{D-2}}{G_{D}}\right)^{1 /(D-2)} S^{\frac{D-3}{D-2}} \sqrt{1+\frac{4 \pi^{2} J^{2}}{S^{2}}} .
$$

The expression for the wavelength estimate $k^{(e s t)}$ we find by equating the entropies is

$$
k^{(e s t)}=k_{s t}^{(e s t)}\left(1+\frac{4 \pi^{2} J^{2}}{S^{2}}\right)^{\frac{D-2}{2(D-3)}}
$$

where

$$
k_{s t}^{(e s t)}=2^{-\frac{D+1}{D-3}} \pi^{-\frac{D-2}{D-3}} \frac{A_{D-3}}{\left(A_{D-2}\right)^{\frac{D-4}{D-3}}} \frac{(D-3)^{D-3}}{(D-2)^{\frac{(D-4)(D-2)}{D-3}}} \frac{1}{\left(G_{D} E\right)^{1 /(D-3)}}
$$

is the wavelength estimate for a static solution with the same value of $E$. From (3.15) we find that nonextremal rotating solutions satisfy the inequality

$$
k_{s t}^{(e s t)} \leq k^{(e s t)}<\left(\frac{D-2}{2}\right)^{\frac{D-2}{2(D-3)}} k_{s t}^{(e s t)}
$$

and thus $k^{(e s t)}$ stays finite in the extremal limit.

The above wavelength estimate can also be expressed in terms of variables used in the numerical procedure as

$$
k^{(e s t)}=2 \pi\left(\frac{D-3}{D-2}\right)^{D-2} \frac{A_{D-3}}{A_{D-2}} \frac{1}{\sqrt{r_{0}^{2}-a^{2}}} .
$$



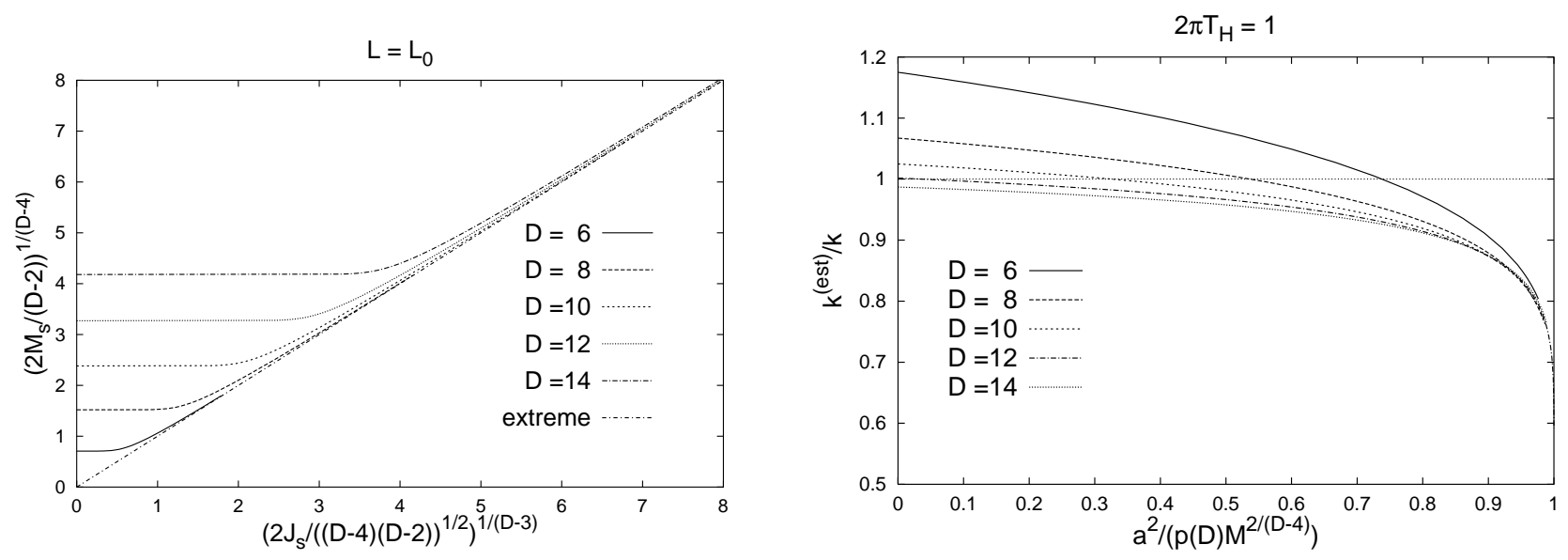

Figure 2: Left: The relation between the scaled mass $M_{s}$ and scaled angular momentum $J_{s}$ for fixed critical length of the extra dimension $L$ is shown for rotating solutions in $D$ even dimensions, $6 \leq D \leq 14$. Both $M_{s}$ and $J_{s}$ are equipped with suitable powers and normalizations. $L_{0}$ represents the value where the instability of the static uniform black string occurs. Right: The ratio betwen the wavelength estimate $k^{(e s t)}$ and the value of $k$ found numerically is plotted at constant temperature $2 \pi T_{H}=1$ as function of the dimensionless quantity $a^{2} /\left(p(D) M^{\frac{2}{D-4}}\right)$.

The fact that, as seen in Figure 1, the numerical value of $k$ takes very large values in the limit $a^{2} /\left(p(D) M^{\frac{2}{D-4}}\right) \rightarrow 1$, is a consequence of keeping the temperature constant and letting $r_{0}$ run. If we would rescale with $r_{0}$, the temperature would vanish in the extreme limit and the rescaled $k$ would stay finite.

The ratio between the numerical value of $k$ one finds by solving the equations $(3.26)$ and the above estimate is presented in Figure 2 (right).

For static black strings, the study of the perturbative equations in second order revealed the appearance of a critical dimension, above which the perturbative nonuniform black strings are less massive than the marginally stable uniform black string [ [ 4 ]. It would therefore be interesting to solve the perturbative equations to second order also in the presence of rotation. But so far we have encountered numerical problems in such an analysis.

\section{4. $D=6$ rotating nonuniform black string solutions}

\subsection{Numerical procedure}

To construct rotating nonuniform black string solutions numerically, we introduce analogous to the static case the new radial coordinate $\tilde{r}$,

$$
\tilde{r}=\sqrt{r^{2}-r_{0}^{2}}
$$

thus the horizon resides at $\tilde{r}=0$. 
The $D=6$ line element Eq. (2.2) then reads (with $\theta_{1}=\theta$ )

$$
\begin{aligned}
d s^{2}= & -e^{2 A(\tilde{r}, z)} \frac{\tilde{r}^{2}}{g(\tilde{r})} d t^{2}+e^{2 B(\tilde{r}, z)}\left(d \tilde{r}^{2}+d z^{2}\right)+e^{2 C(\tilde{r}, z)} g(\tilde{r}) d \theta^{2} \\
& +e^{2 G(\tilde{r}, z)} g(\tilde{r})\left(\sin ^{2} \theta\left(d \varphi_{1}-W(\tilde{r}, z) d t\right)^{2}+\cos ^{2} \theta\left(d \varphi_{2}-W(\tilde{r}, z) d t\right)^{2}\right) \\
& -\left(e^{2 G(\tilde{r}, z)}-e^{2 C(\tilde{r}, z)}\right) g(\tilde{r}) \sin ^{2} \theta \cos ^{2} \theta\left(d \varphi_{1}-d \varphi_{2}\right)^{2},
\end{aligned}
$$

where $g(\tilde{r})=r_{0}^{2}+\tilde{r}^{2}$.

We then change to dimensionless coordinates $\rho$ and $\zeta$,

$$
\rho=\tilde{r} /\left(r_{0}+\tilde{r}\right), \quad \zeta=z / L,
$$

where the compactified radial coordinate $\rho$ maps spatial infinity to the finite value $\rho=1$, and $L$ is the asymptotic length of the compact direction.

We solve the resulting set of five coupled non-linear elliptic partial differential equations numerically, subject to the boundary conditions Eqs. (2.13)-(2.15). These numerical calculations are based on the Newton-Raphson method and are performed with help of the program FIDISOL [36], which provides also an error estimate for each unknown function.

The equations are discretized on a non-equidistant grid in $\rho$ and $\zeta$. Typical grids used have sizes $65 \times 50$, covering the integration region $0 \leq \rho \leq 1$ and $0 \leq \zeta \leq 1 / 2$. (See [36] and [37] for further details and examples for the numerical procedure.) For the nonuniform strings the estimated relative errors range from approximately $\approx 0.001 \%$ for small geometric deformation to $\approx 1 \%$ for large deformation. We also monitored the violation of the weighted constraints $\sqrt{f} \sqrt{-g}\left(G_{r}^{r}-G_{z}^{z}\right)=0$, and $\sqrt{-g} G_{z}^{r}=0$, which is typically less then 0.1.

The horizon coordinate $r_{0}$ and the asymptotic length $L$ of the compact direction enter the equations of motion as parameters. The results presented are obtained with the parameter choice

$$
r_{0}=1, \quad L=L^{\text {crit }}=4.9516,
$$

where $L^{\text {crit }}$ represents the value, where the instability of the static uniform black string occurs.

Rotating nonuniform black strings can then be obtained by starting from a static nonuniform black string solution and increasing the value of angular velocity $\Omega_{H}$ of the event horizon, which enters the boundary conditions. By varying also the second boundary parameter $d_{0}$, associated with the temperature of the black strings, $d_{0}=\ln \left(T_{H}^{(0)} / T_{H}\right)$ (see Eq. (2.21)), the full set of rotating nonuniform black strings can then be explored. An alternative procedure to obtain rotating NUBS numerically would be, to start from stationary perturbative nonuniform solutions.

The basic properties of the NUBS are encoded in the five metric functions $A(\rho, \zeta), B(\rho, \zeta)$, $C(\rho, \zeta), G(\rho, \zeta)$, and $W(\rho, \zeta)$. These functions change smoothly with the two boundary parameters $d_{0}$ and $\Omega_{H}$. We illustrate these functions in Figure 3, for the parameter choices $d_{0}=0.6$ and $\Omega_{H}=0.25$ resp. $\Omega_{H}=0.202$, the latter corresponding to a solution in the strongly deformed region close to the expected transition from rotating nonuniform black strings to rotating caged black holes. 

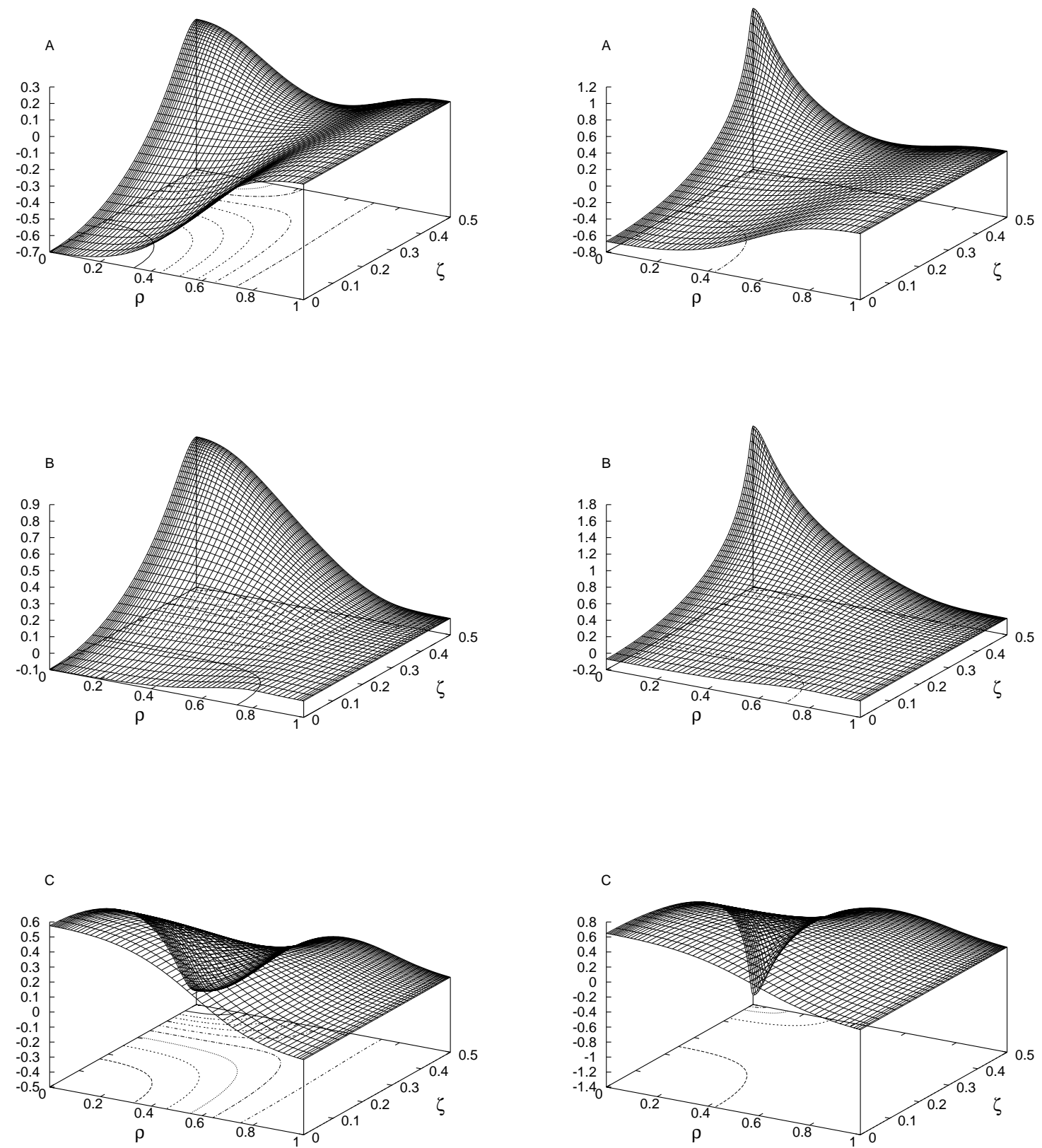

Figure 3: The metric functions $A, B, C, G$ and $W$ of the $D=6$ rotating nonuniform black string solution with temperature parameter $d_{0}=0.6$ and horizon angular velocity $\Omega_{H}=0.25$ (left column) and $\Omega_{H}=0.202$ (right column) are shown as functions of the compactified radial coordinate $\rho$, and the coordinate $\zeta$ of the compact direction. Note that the horizon is located at $\rho=0$. 
G
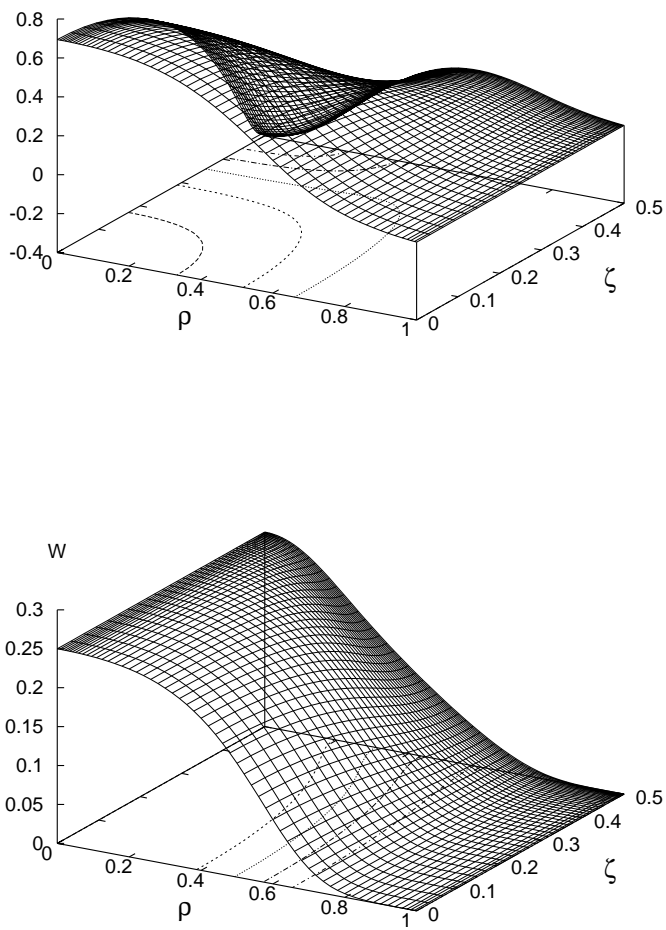

G
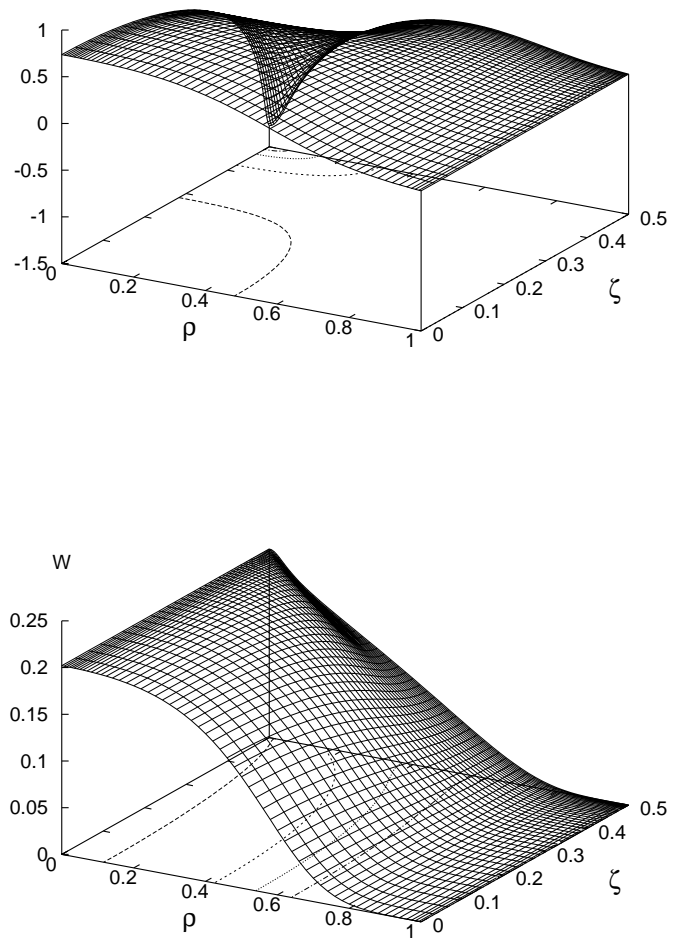

Figure 3: continued.

\subsection{Properties of rotating black strings}

\subsubsection{The horizon}

For the static NUBS a measure of their deformation is given by the nonuniformity parameter $\lambda[2]$

$$
\lambda=\frac{1}{2}\left(\frac{\mathcal{R}_{\max }}{\mathcal{R}_{\min }}-1\right)
$$

where $\mathcal{R}_{\max }$ and $\mathcal{R}_{\text {min }}$ represent the maximum radius of a $(D-3)$-sphere on the horizon and the minimum radius, respectively, the minimum radius being the radius of the 'waist' of the black string. Thus for uniform black strings $\lambda=0$, while the horizon topology changing transition should be approached for $\lambda \rightarrow \infty$ [38, 39].

For the rotating NUBS one has to take into account, that the rotation leads to a deformation of the 3-sphere of the horizon, making it oblate w.r.t. the planes of rotation. Therefore, various possibilities arise to define the nonuniformity parameter $\lambda$. In the following we em- 
ploy the above definition of $\lambda$, where $\mathcal{R}_{\max }$ and $\mathcal{R}_{\text {min }}$ are obtained from the area $A_{H}$ of the respective deformed 3 -sphere via $A_{H}=2 \pi^{2} \mathcal{R}^{3}$.

In Figure 4 we exhibit the spatial embedding of the horizon into 3-dimensional space for a sequence of $D=6$ rotating NUBS. In these embeddings the symmetry directions $\left(\varphi_{1}, \varphi_{2}\right)$ are suppressed, and the proper circumference of the horizon is plotted against the proper length along the compact direction, yielding a geometrical view of both the deformation of the horizon due to rotation and the nonuniformity of the horizon with respect to the compact coordinate.

For the solutions of the sequence shown in Figure 4 the temperature is kept fixed with temperature parameter $d_{0}=0.6$. The first solution of the sequence corresponds to the marginally stable rotating uniform black string, which has $\lambda=0$ and horizon angular velocity $\Omega_{H}=0.34908$. When the horizon angular velocity is lowered, rotating black strings with increasing nonuniformity are obtained. Shown are solutions with nonuniformity parameter $\lambda=0.83,1.7$ and 2.9. The latter is already close to the expected topology changing transition to rotating caged black holes. Interestingly, close to the maximal radius $\mathcal{R}_{\text {max }}$ the deformation of the horizon due to rotation is significant, whereas close to $\mathcal{R}_{\min }$ the 3 -horizon appears to be almost spherical.

The deformation of the horizon due to rotation is demonstrated in more detail in Figure 5 , where circumferences of the deformed 3 -sphere of the horizon are exhibited. Here $l_{\mathrm{e}, \max }$ denotes the equatorial maximum circumference, and $l_{\mathrm{e}, \mathrm{min}}$ the equatorial minimum circumference, both referring to the circumferences in the two equivalent planes of rotation, while $l_{\mathrm{p}, \max }$ denotes the polar maximum circumference, and $l_{\mathrm{p}, \mathrm{min}}$ the polar minimum circumference, representing circumferences for fixed azimuthal angles. In the static case, the respective equatorial and polar circumferences agree, and the minimum circumference represents the circumference of the waist of the NUBS.

Using the scaled energy-mass $M_{s}$ and the scaled angular momentum $J_{s}$, Eq. (3.28), we exhibit in Figure 5 these polar and equatorial circumferences versus the scaled angular momentum ratio $J_{s} / M_{s}^{3 / 2}$. We note, that for rotating uniform black strings, this ratio is bounded, $J_{s} / M_{s}^{3 / 2} \leq 1$, with extremal rotating uniform solutions saturating the bound.

For reference, the figure exhibits the polar and equatorial circumferences of the branch of marginally stable MP uniform black strings. This uniform branch ranges from the static marginally stable black string to the extremal rotating marginally stable black string. The static marginally stable string, with all circumferences equal, has temperature parameter $d_{0}=0$. Along this rotating UBS branch, with equal maximal and minimal circumferences, the temperature parameter $d_{0}$ and the deformation of the horizon 3-sphere due to rotation both increase monotonically, while the temperature itself decreases monotonically, reaching zero in the extremal case.

\subsubsection{A critical temperature}

The rotating nonuniform black strings branch off from the marginally stable uniform black strings. These branches are obtained, by fixing a value of the temperature parameter $d_{0}$ 


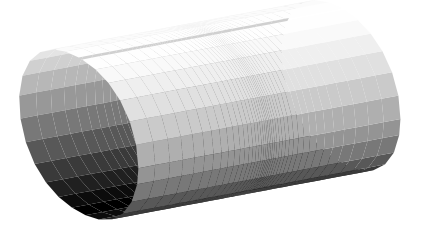

$$
\begin{aligned}
& \Omega_{H}=0.34908 \\
& \lambda=0
\end{aligned}
$$
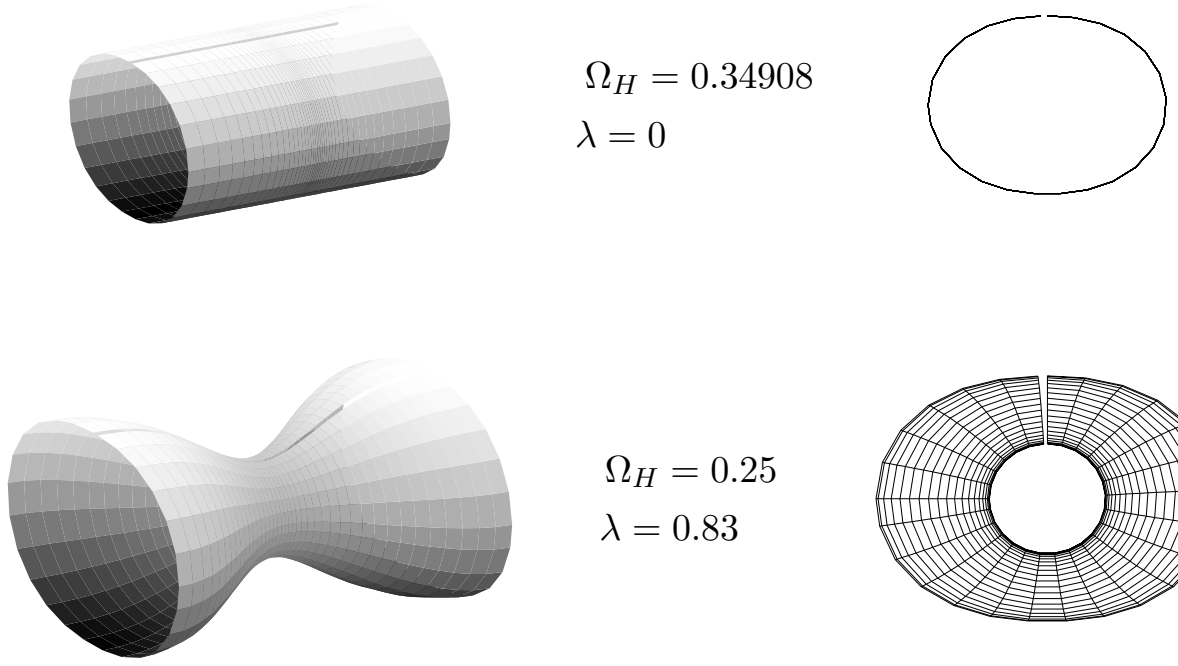

$$
\begin{aligned}
& \Omega_{H}=0.25 \\
& \lambda=0.83
\end{aligned}
$$
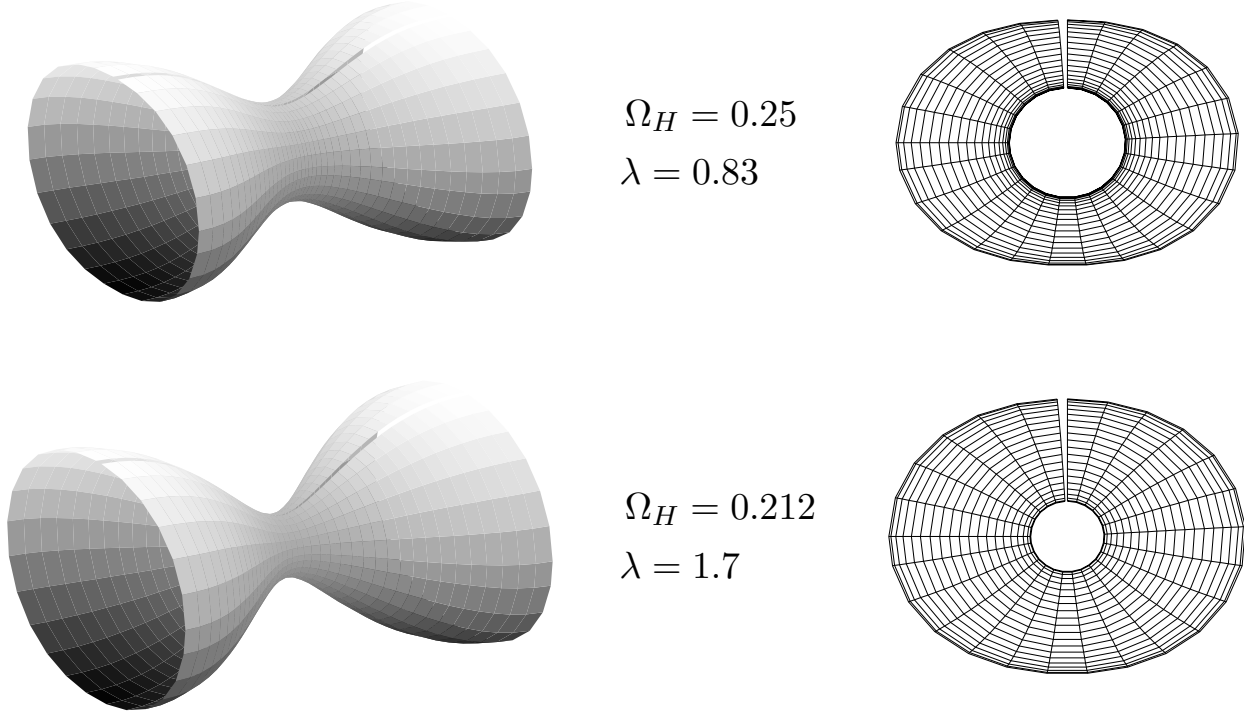

$$
\Omega_{H}=0.212
$$$$
\lambda=1.7
$$
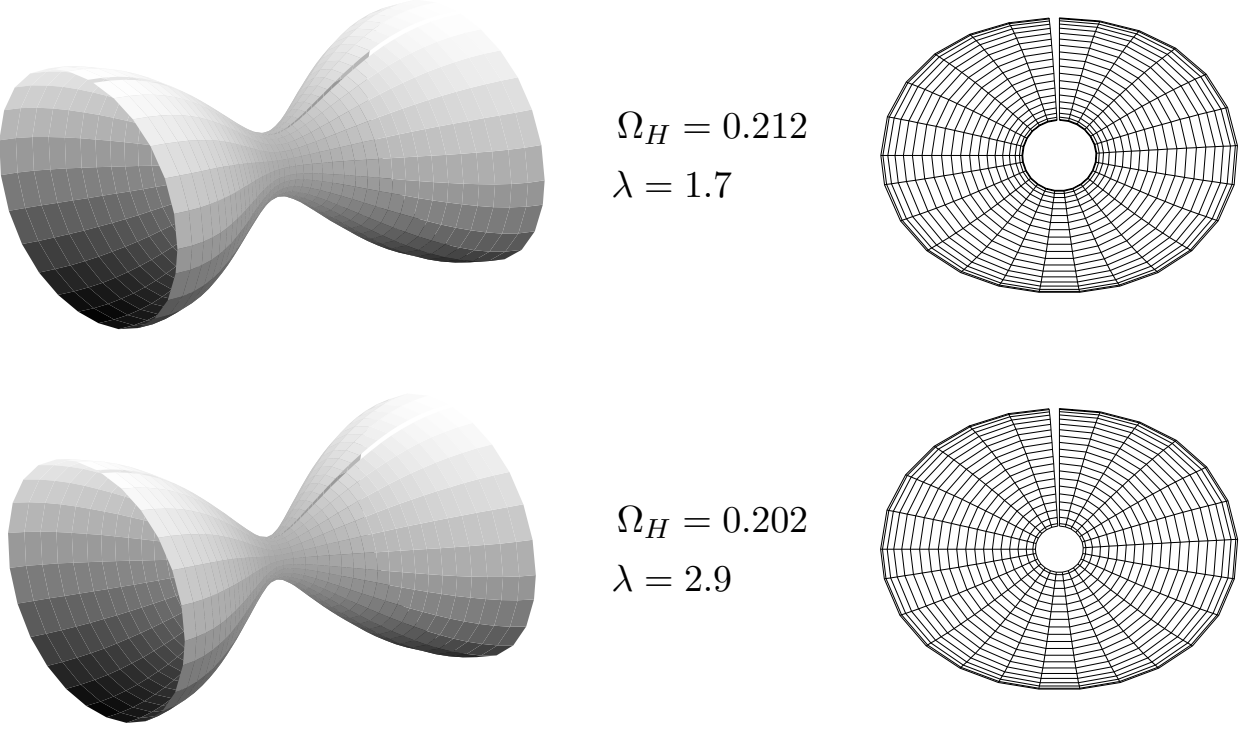

$$
\begin{aligned}
& \Omega_{H}=0.202 \\
& \lambda=2.9
\end{aligned}
$$

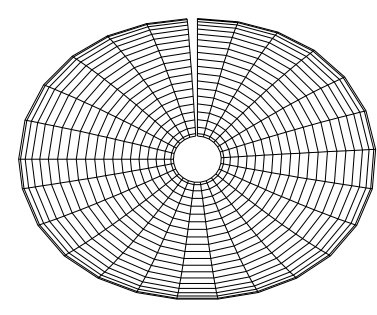

Figure 4: The spatial embedding of the horizon of $D=6$ rotating black string solutions is shown for a sequence of solutions with fixed temperature parameter $d_{0}=0.6$ and varying horizon angular velocity $\Omega_{H}: \Omega_{H}=0.34908$ (upper row), $\Omega_{H}=0.25$ (second row), $\Omega_{H}=0.212$ (third row) and $\Omega_{H}=0.202$ (lower row), $\lambda$ specifies the increasing nonuniformity of the solutions. Left column: side view, right column: view in $z$ direction. $\left(r_{0}=1, L=L^{\text {crit }}=4.9516\right.$. $)$ 
and thus fixing the temperature, and then decreasing the horizon angular velocity $\Omega_{H}$ from the respective rotating UBS value. Depending on the value of the fixed chosen temperature parameter $d_{0}$, the corresponding rotating NUBS branches exhibit distinct features. When $d_{0}<d_{0}^{*}$, the rotating NUBS branch extends back to a static NUBS solution with a finite waist, and thus finite minimal circumferences $l_{\mathrm{p}, \min }$ and $l_{\mathrm{e}, \mathrm{min}}$. The size of the waist of the static NUBS solution decreases with increasing $d_{0}$. At the critical value $d_{0}^{*}$, the respective branch of rotating NUBS is expected to extend precisely back to a static solution with zero size waist, i.e., to the solution at the topology changing transition, where the branch of static NUBS merges with the branch of static caged black holes. This critical value of the temperature parameter $d_{0}^{*}$ is in the interval $0.30<d_{0}<0.33$, and corresponds to a critical value of the temperature $T_{*}$ where $0.72<T_{H} / T_{0}<0.74$ (with $T_{0}$ the temperature of the UBS). This may be compared with our previous results for static black strings [5]. Extraction of the critical temperature $T_{*}$ from those static black string calculations, suggests the bounds $0.72<T_{H} / T_{0}<0.76$ for the critical temperature $T_{*}$ (when trying to account for numerical inaccuracy in the critical region). ${ }^{3}$

Beyond the critical value $d_{0}^{*}$, the branches of rotating NUBS no longer reach static NUBS. Instead they are expected to extend to a corresponding rotating solution with zero size waist, and thus to lead towards a topology changing transition, associated with the merging of a branch of rotating NUBS and a branch of rotating caged black holes. Indeed, when $d_{0}>d_{0}^{*}$, the waist of the NUBS solutions monotonically decreases in size, the minimal circumferences approaching zero. Thus we see here first evidence, that a topology changing transition arises also for rotating branches of solutions. We note, that the deformation of the horizon 3sphere due to rotation is considerable at maximum size, while the waist of the rotating NUBS becomes increasingly spherical.

In Figure 6 we exhibit the nonuniformity parameter $\lambda$ versus the scaled angular momentum ratio $J_{s} / M_{s}^{3 / 2}$ for the same set of rotating NUBS. The branches begin at the rotating marginally stable UBS with $\lambda=0$. When $d_{0}<d_{0}^{*}$, the rotating NUBS branches extend back to static NUBS solutions with finite nonuniformity and thus finite waist. When $d_{0}>d_{0}^{*}$, on the other hand, the nonuniformity parameter $\lambda$ increases apparently without bound, approaching the topology changing transition for $\lambda \rightarrow \infty$.

The branch of rotating marginally stable UBS is bounded by the static and by the extremal rotating solution. It would be interesting to obtain the corresponding domain of existence of rotating NUBS. The construction of extremal rotating NUBSs (if they exist), however, currently represents an unsolved numerical challenge.

Figure 6 also exhibits the scaled horizon angular velocity $\Omega_{H} / \Omega_{H, \mathrm{GL}}$ for this set of rotating NUBS versus the scaled angular momentum ratio $J_{s} / M_{s}^{3 / 2}$. Here $\Omega_{H, \mathrm{GL}}$ denotes the horizon angular velocity of the marginally stable rotating UBS (with the same temperature parameter $\left.d_{0}\right)$. Starting from rotating marginally stable UBS with $\Omega_{H} / \Omega_{H, \mathrm{GL}}=1$, the branches end at

\footnotetext{
${ }^{3}$ Such an identification of $T_{*}$ assumes a monotonic dependence of the temperature of the static uniform strings on the nonuniformity parameter $\lambda$, since otherwise bifurcations might be present and complicate this scenario.
} 

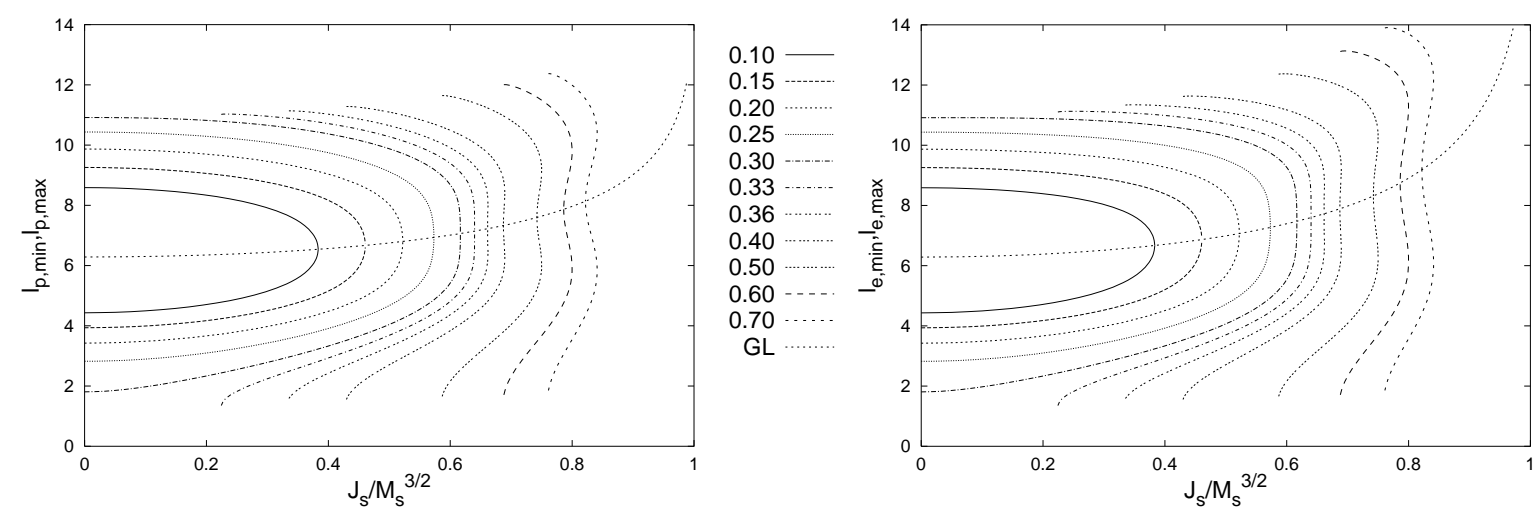

Figure 5: The maximum and minimum polar circumferences $l_{\mathrm{p}, \max }$ and $l_{\mathrm{p}, \min }$ of the deformed horizon 3 -sphere, and the respective equatorial circumferences $l_{\mathrm{e}, \max }$ and $l_{\mathrm{e}, \min }$ are shown versus the scaled angular momentum ratio $J_{s} / M_{s}^{3 / 2}$ for branches of rotating NUBS with fixed values of the temperature parameter $d_{0}$ ranging from 0.1 to 0.7 . The circumferences of the deformed horizon 3 -sphere are also shown for marginally stable MP UBS (denoted GL).

static NUBS with $\Omega_{H}=J=0$, when $d_{0}<d_{0}^{*}$. When $d_{0}>d_{0}^{*}$, in contrast, the branches of rotating NUBS appear to approach limiting solutions with finite horizon angular velocity $\Omega_{H}$ and finite angular momentum $J$, associated with a topology changing transition.
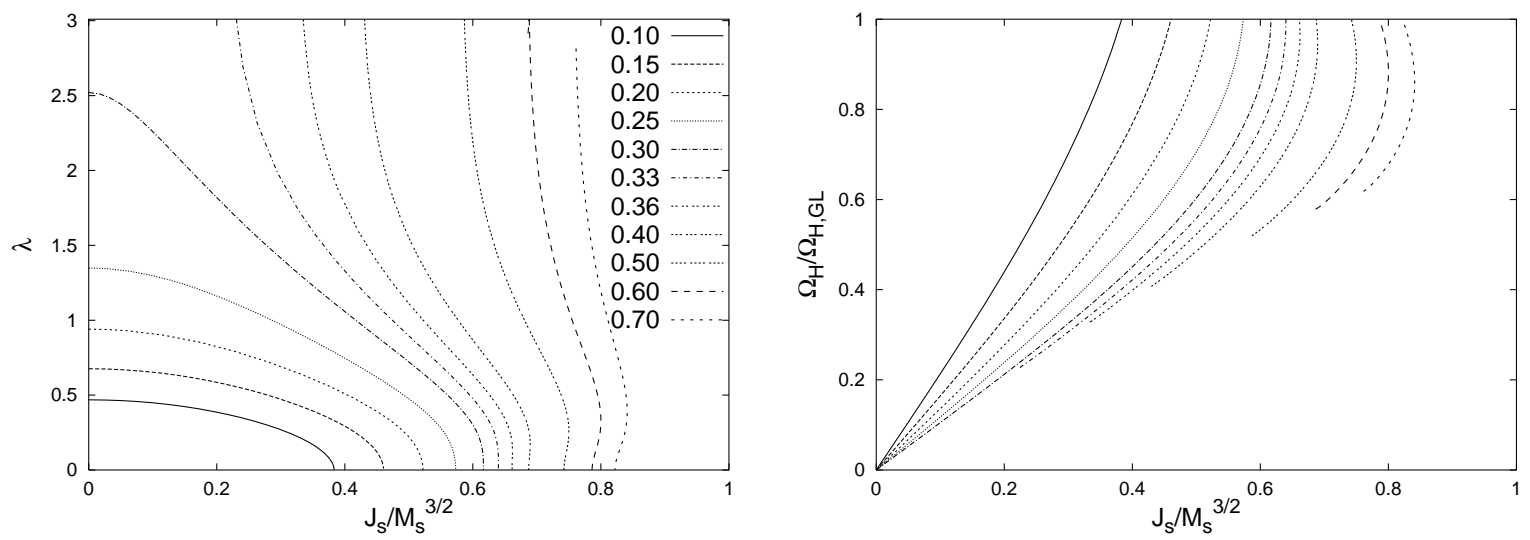

Figure 6: The nonuniformity parameter $\lambda$ and the scaled horizon angular velocity $\Omega_{H} / \Omega_{H, \mathrm{GL}}$ are shown versus the scaled angular momentum ratio $J_{s} / M_{s}^{3 / 2}$ for branches of rotating NUBS with fixed values of the temperature parameter $d_{0}$ ranging from 0.1 to 0.7 . ( $\Omega_{H, \mathrm{GL}}$ denotes the angular velocity of the marginally stable MP UBS.)

\subsubsection{Global charges}

The scaled mass $M_{s} / M_{s, \mathrm{GL}}$ and the scaled entropy $S_{s} / S_{s, \mathrm{GL}}$ (where $S=S_{s} A_{D-3} L / 4$ ) are 
exhibited in Figure 7 versus the scaled angular momentum ratio $J_{s} / M_{s}^{3 / 2}$ for the same set of solutions. Both $M_{s} / M_{s, \mathrm{GL}}$ and $S_{s} / S_{s, \mathrm{GL}}$ increase monotonically along the branches of solutions with fixed temperature. As noted above, when $T_{H}>T_{*}$, the branches of rotating NUBS end at static NUBS, whereas, when $T_{H}<T_{*}$, they appear to approach rotating limiting solutions with finite values of the angular momentum $J$, associated with a topology changing transition between branches of rotating solutions. We conclude from the figure, that the scaled mass $M_{s} / M_{s, \mathrm{GL}}$ of the limiting solutions increases with increasing $J_{s} / M_{s}^{3 / 2}$, while their scaled entropy $S_{s} / S_{s, \text { GL }}$ appears to be almost constant.
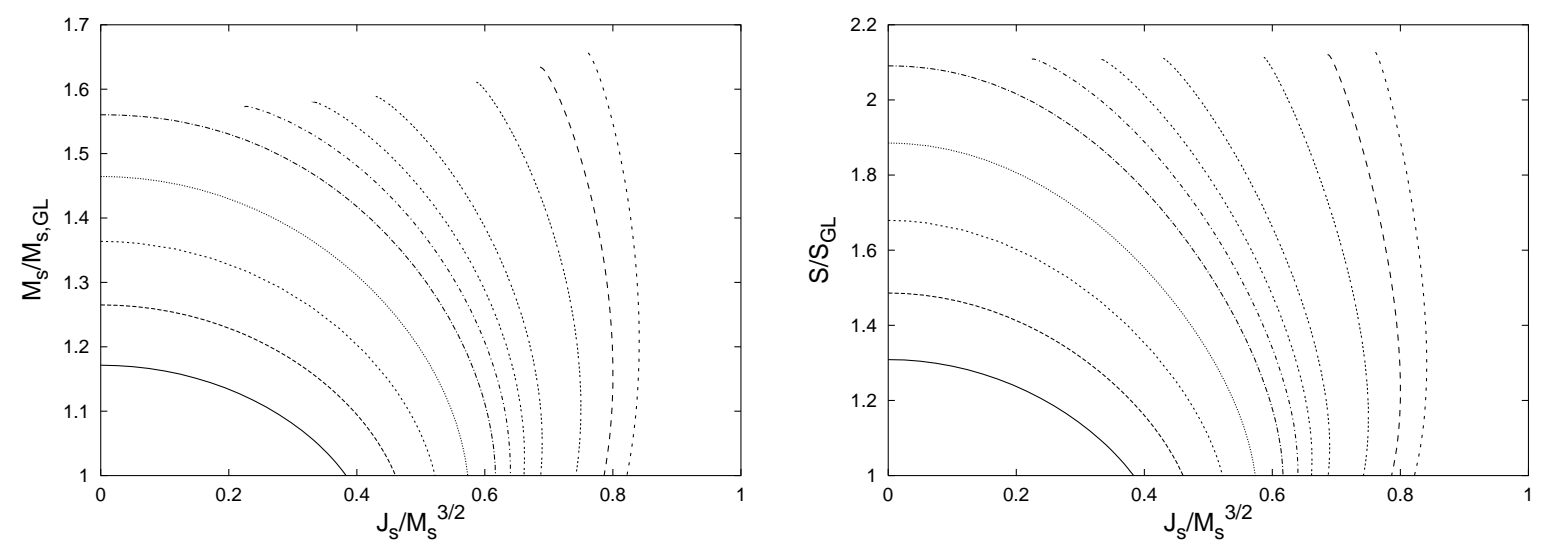

Figure 7: Same as Figure 6 for the scaled mass $M_{s} / M_{s, \mathrm{GL}}$ and the scaled entropy $S_{s} / S_{s, \mathrm{GL}}$. ( $M_{s, \mathrm{GL}}$ and $S_{s, \mathrm{GL}}$ denote the respective quantities of the marginally stable MP UBS.)

We exhibit the relative tension $n$ of this set of rotating NUBS in Figure 8, together with the relative tension of the uniform black strings, $n_{\mathrm{GL}}=1 / 3$. Starting from rotating marginally stable UBS, the relative tension $n$ decreases monotonically for branches of rotating NUBS with large values of the temperature. As the critical temperature $T_{*}$ is approached, and beyond the critical temperature, the tension $n$ no longer decreases monotonically, but instead reaches a minimum and then increases again. Thus we observe the backbending phenomenon for the relative tension $n$, encountered previously for static NUBS [5], also for rotating nonuniform black strings. For the static NUBS we obtained for the relative tension $n$ the critical value $n_{*} \approx 0.2$. Consistency requires, that this value agrees within error bounds with the critical value obtained here for the branch of rotating NUBS at the critical temperature $T_{*}$. The figure indicates, that this requirement may hold.

Restricting to a canonical ensemble, the numerical analysis indicates that the qualitative thermodynamical features of the uniform MP branch are also shared by rotating NUBS solutions. For small values of $J$, the entropy is a decreasing function of $T$, i.e. $C_{J, L}<0$. However, the configurations near extremality are thermally stable in a canonical ensemble. Also, although further work is necessary in this case, we expect all nonuniform solutions to be thermodynamically unstable in a grand canonical ensemble. 


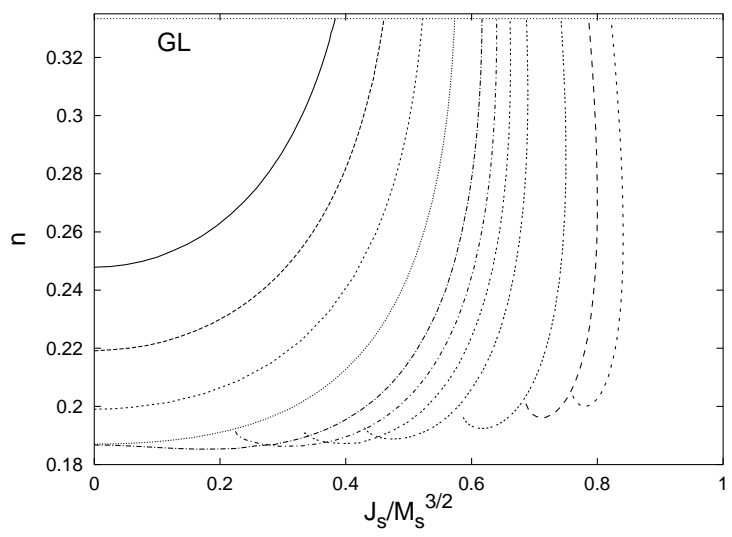

Figure 8: Same as Figure 6 for the relative tension $n$.

\subsubsection{The ergoregion}

Like rotating black holes, rotating black strings possess an ergoregion. While the ergosurface of rotating uniform black strings is uniform like the horizon, the ergosurface of nonuniform black strings reflects the nonuniformity of the horizon. In Figure 9 we exhibit the spatial embedding of the ergosurface into 3-dimensional space for those rotating NUBS, whose spatial embedding of the horizon was shown in Figure 4.

As in Figure 4, the symmetry directions are suppressed here. The proper circumference of the ergosurface is plotted against the proper arclength along the compact direction. Denoting $\tilde{r}_{e}(z), z$ the coordinates of the ergosurface at fixed $\varphi_{1}, \varphi_{2}, \theta, t$, we define the arclength as

$$
\sigma=\int_{0}^{z} e^{B\left(\tilde{r}_{e}(z), z\right)} \sqrt{1+\left(\frac{d \tilde{r}_{e}}{d z}\right)^{2}} d z
$$

The solutions shown have fixed temperature with temperature parameter $d_{0}=0.6$, decreasing horizon angular velocity $\Omega_{H}$, and increasing nonuniformity of the horizon. The first solution corresponds to the marginally stable rotating uniform black string, with an ergosurface uniform w.r.t. the compact coordinate, but rotational deformation. When the horizon angular velocity is lowered, the nonuniformity of the ergosurface increases, along with the increase of the nonuniformity of the horizon.

In Figure 10 we exhibit the ergosurface in terms of the coordinates $r_{\text {ergo }}=\tilde{r}$ and $\zeta$, in which the horizon is located at $\tilde{r}=0$. We show rotating NUBS solutions on three branches with fixed temperature, corresponding to the values of the temperature parameter $d_{0}=0.3$, 0.33 and 0.6. Thus the first two sets represent solutions just above and just below the critical temperature $T_{*}$. All sets start with the corresponding rotating UBS and thus a uniform ergosurface. As the horizon angular velocity $\Omega_{H}$ decreases from $\Omega_{H, \mathrm{GL}}$ nonuniformity of the ergosurface develops. 


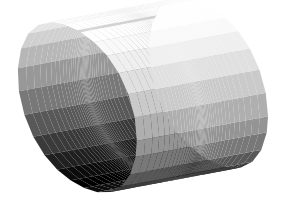

$$
\begin{aligned}
& \Omega_{H}=0.34908 \\
& \lambda=0
\end{aligned}
$$
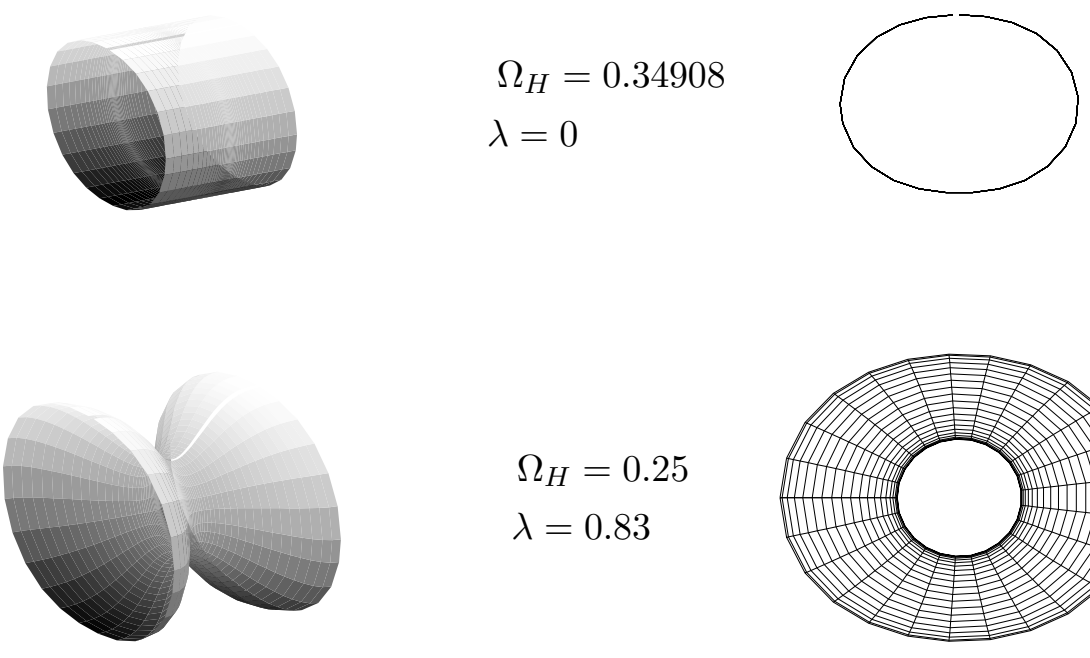

$$
\begin{aligned}
& \Omega_{H}=0.25 \\
& \lambda=0.83
\end{aligned}
$$
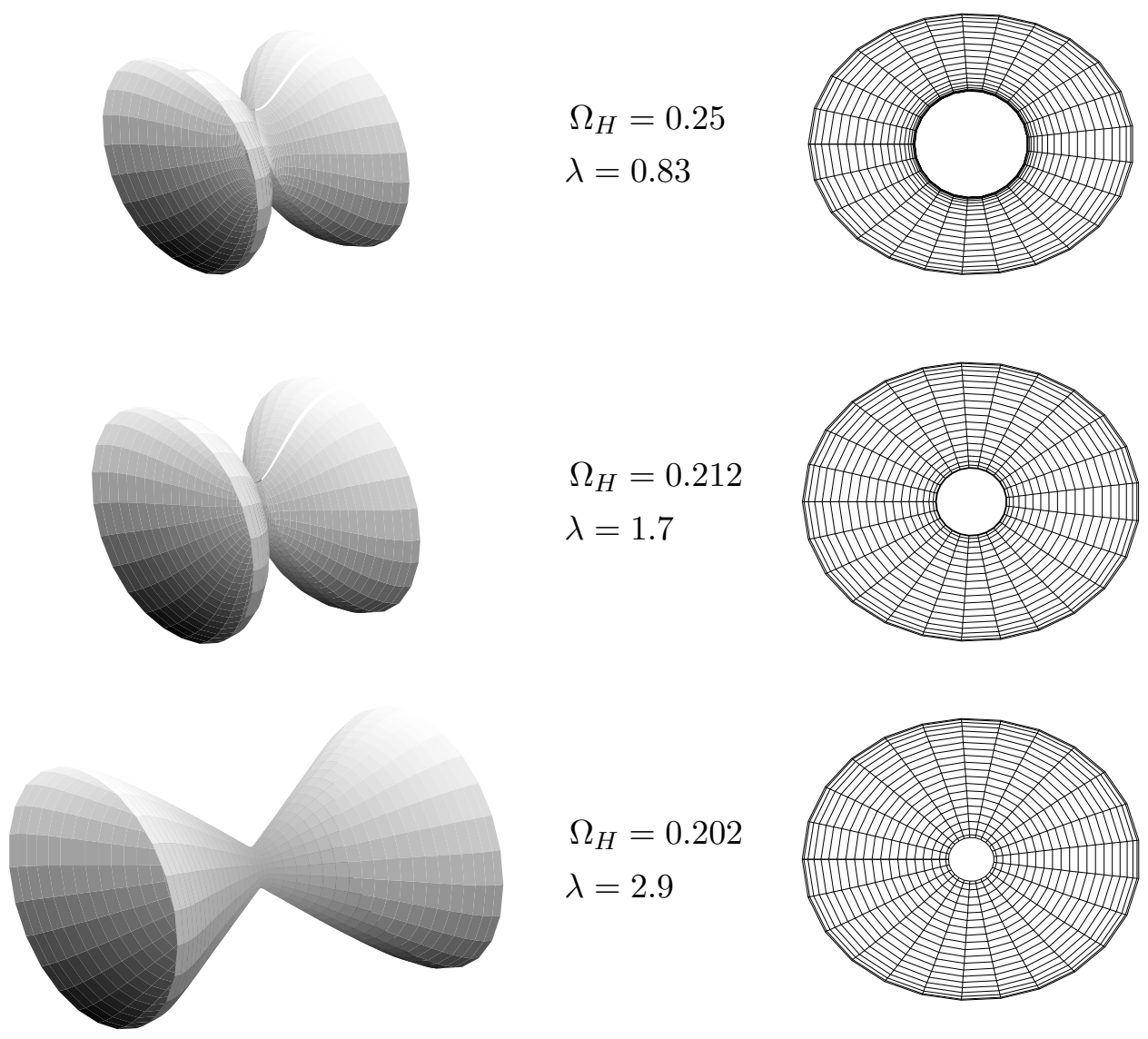

$$
\begin{aligned}
& \Omega_{H}=0.212 \\
& \lambda=1.7
\end{aligned}
$$$$
\Omega_{H}=0.202
$$$$
\lambda=2.9
$$

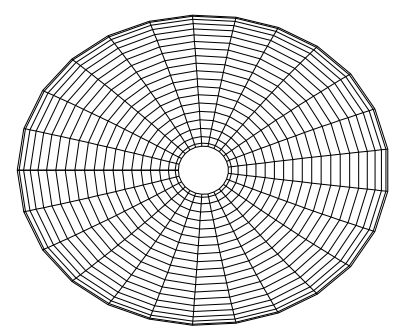

Figure 9: The spatial embedding of the ergosurface. of $D=6$ rotating black string solutions is shown for a sequence of solutions with fixed temperature parameter $d_{0}=0.6$ and varying horizon angular velocity $\Omega_{H}: \Omega_{H}=0.34908$ (upper row), $\Omega_{H}=0.25$ (second row), $\Omega_{H}=0.212$ (third row) and $\Omega_{H}=0.202$ (lower row), $\lambda$ specifies the increasing nonuniformity of the solutions. Left column: side view, right column: view in $z$ direction. $\left(r_{0}=1, L=L^{\text {crit }}=4.9516\right.$. $)$ 

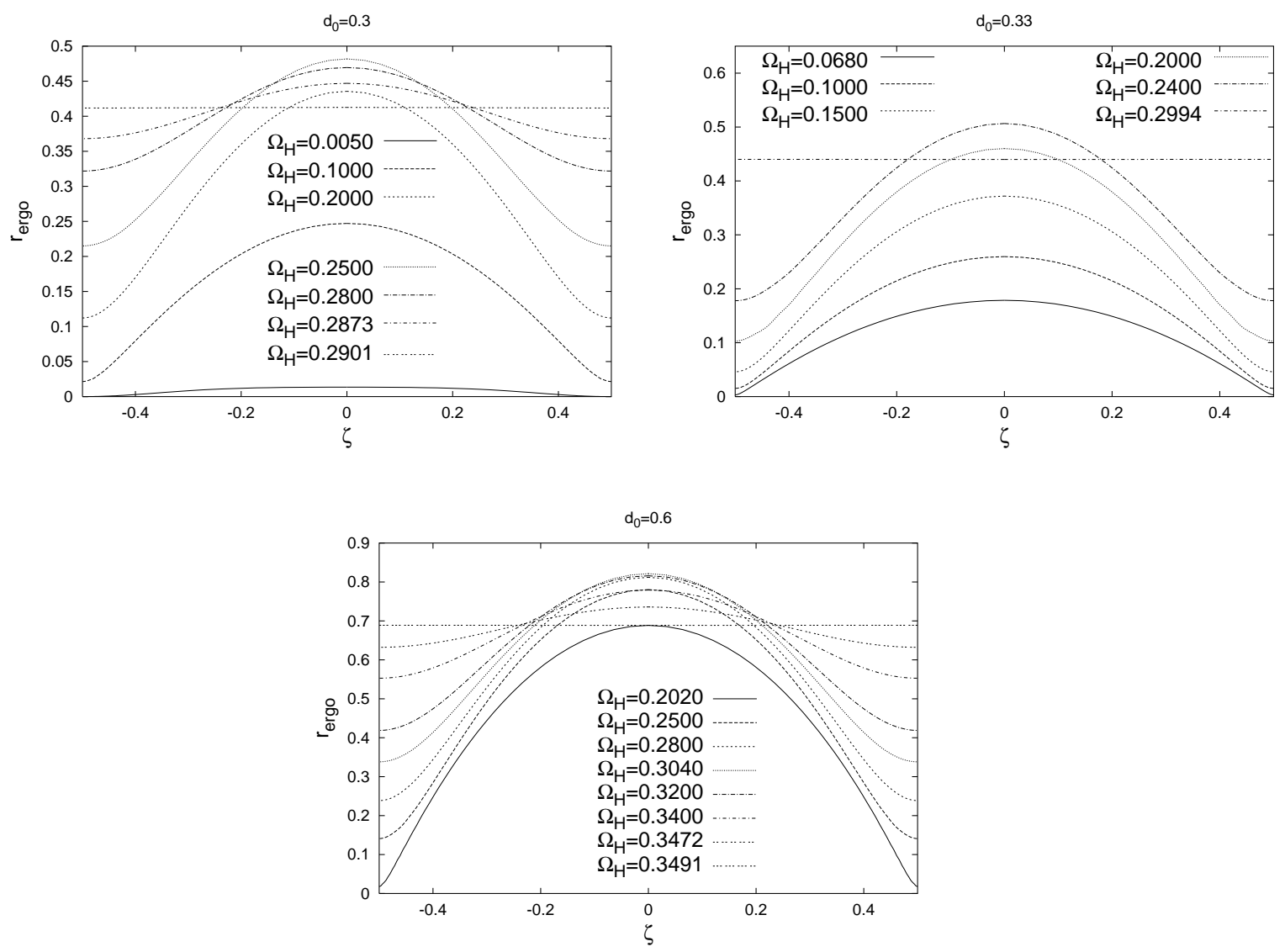

Figure 10: The ergosurface of rotating NUBS is shown for three sets of solutions with fixed temperature, corresponding to the values of the temperature parameter, $d_{0}=0.3,0.33$ and 0.6 , and decreasing horizon angular velocity $\Omega_{H}$, starting from the value $\Omega_{H, \mathrm{GL}}$ of the respective marginally stable MP UBS.

For $d_{0}=0.3$, where $T_{H}>T_{*}$, the rotating NUBS branch extends back to a static NUBS solution, where the ergoregion disappears. The strong shrinkage of the ergoregion close to this point is clearly seen in the figure for the NUBS solution with horizon angular velocity $\Omega_{H}=0.005$. For $d_{0}=0.33$, the temperature is just below the critical value, $T_{H}<T_{*}$, and the rotating NUBS branch is expected to extend to a corresponding rotating solution with zero size waist, and thus signify a topology changing transition between rotating solutions. This is reflected in the figure by the presence of a finite ergoregion of the solution with $\Omega_{H}=0.068$, close to the transition point. Also for $d_{0}=0.6$, the ergoregion remains finite, as the topology changing transition is approached. We note, that the size of the ergoregion of the limiting solution appears to increase with decreasing temperature. 


\section{Rotating NUBS in heterotic string theory}

In order to obtain rotating electrically charged black strings, we employ a solution generating technique, by performing symmetry transformations on the neutral solution. Within toroidally compactified heterotic string, an approach to obtain the charged solutions from the neutral one was presented in Ref. [41. This method was used to obtain, e.g., general rotating electrically charged solutions in four dimensions [41], higher-dimensional general electrically charged static solutions [42], and rotating black hole solutions with one rotational parameter in $D$ dimensions 43 . General $D$-dimensional charged rotating black holes with $[(D-1) / 2]$ distinct angular momenta were constructed in 44.

The massless fields in heterotic string theory compactified on a $(10-D)$-dimensional torus consist of the string metric $G_{\mu \nu}$, the anti-symmetric tensor field $B_{\mu \nu},(36-2 D) \mathrm{U}(1)$ gauge fields $A_{\mu}^{(j)}(1 \leq j \leq 36-2 D)$, the scalar dilaton field $\Phi$, and a $(36-2 D) \times(36-2 D)$ matrix valued scalar field $M$ satisfying,

$$
M L M^{T}=L, \quad M^{T}=M .
$$

Here $L$ is a $(36-2 D) \times(36-2 D)$ symmetric matrix with $(26-D)$ eigenvalues -1 and $(10-D)$ eigenvalues +1 . Following [41], [42] we shall take $L$ to be

$$
L=\left(\begin{array}{cc}
-I_{26-D} & \\
& I_{10-D}
\end{array}\right)
$$

where $I_{n}$ denotes an $n \times n$ identity matrix. The action describing the effective field theory of these massless bosonic fields is given by 40

$$
\begin{aligned}
S= & \int d^{D} x \sqrt{-\operatorname{det} G} e^{-\Phi}\left[R_{G}+G^{\mu \nu} \partial_{\mu} \Phi \partial_{\nu} \Phi+\frac{1}{8} G^{\mu \nu} \operatorname{Tr}\left(\partial_{\mu} M L \partial_{\nu} M L\right)\right. \\
& \left.-\frac{1}{12} G^{\mu \mu^{\prime}} G^{\nu \nu^{\prime}} G^{\rho \rho^{\prime}} H_{\mu \nu \rho} H_{\mu^{\prime} \nu^{\prime} \rho^{\prime}}-G^{\mu \mu^{\prime}} G^{\nu \nu^{\prime}} F_{\mu \nu}^{(j)}(L M L)_{j k} F_{\mu^{\prime} \nu^{\prime}}^{(k)}\right],
\end{aligned}
$$

where

$$
\begin{gathered}
F_{\mu \nu}^{(j)}=\partial_{\mu} A_{\nu}^{(j)}-\partial_{\nu} A_{\mu}^{(j)} \\
H_{\mu \nu \rho}=\partial_{\mu} B_{\nu \rho}+2 A_{\mu}^{(j)} L_{j k} F_{\nu \rho}^{(k)}+\text { cyclic permutations of } \mu, \nu, \rho,
\end{gathered}
$$

and $R_{G}$ denotes the scalar curvature associated with the metric $G_{\mu \nu}$. The canonical Einstein metric $\bar{g}_{\mu \nu}$ is

$$
\bar{g}_{\mu \nu}=e^{-\frac{2}{D-2} \Phi} G_{\mu \nu} .
$$

One can add a general charge to any stationary vacuum solution by applying the solution generating transformations $(O(26-D, 1) / O(26-D)) \times(O(10-D, 1) / O(10-D))$. This generates a nontrivial $\Phi, B_{\mu \nu}$ and $M$, as well as $A_{\mu}^{(j)}$. 
Here we are mainly interested in the case $D=6$, i.e., heterotic string theory compactified on a four-dimensional torus. Since the generation procedure is identical to the one given in Ref.41] we shall not give the details, but only present the final results.

Starting with an arbitrary time independent solution $g_{\mu \nu}$ of the vacuum field equation in $D=6$ (with $d s^{2}=g_{\mu \nu} d x^{\mu} d x^{\nu}$ ), the expression for the metric of a charged configuration expressed in the canonical Einstein frame is

$$
\begin{aligned}
& d \bar{s}^{2}=\Delta^{1 / 4}\left(d s^{2}+\frac{1}{g_{t t}}\left(\frac{(\cosh \alpha+\cosh \beta)^{2}}{4 \Delta}-1\right)\left(g_{\varphi_{1} t} d \varphi_{1}+g_{\varphi_{2} t} d \varphi_{2}\right)^{2}\right. \\
& \left.+\left(\frac{\cosh \alpha+\cosh \beta}{\Delta}-2\right)\left(g_{\varphi_{1} t} d \varphi_{1} d t+g_{\varphi_{2} t} d \varphi_{2} d t\right)+\left(\frac{1-\Delta}{\Delta}\right) g_{t t} d t^{2}\right),
\end{aligned}
$$

where $\Delta$ is related to the dilaton,

$$
e^{-2 \Phi}=\Delta=\frac{1}{4}\left(g_{t t}^{2}(\cosh \alpha-\cosh \beta)^{2}+2 g_{t t}\left(\sinh ^{2} \alpha+\sinh ^{2} \beta\right)+(\cosh \alpha+\cosh \beta)^{2}\right)
$$

The time components of the $U(1)$ gauge fields are

$$
\begin{aligned}
A_{t}^{(i)} & =-\frac{n^{(i)}}{4 \sqrt{2} \Delta}\left(1+g_{t t}\right) \sinh \alpha\left(\cosh \alpha+\cosh \beta+g_{t t}(\cosh \alpha-\cosh \beta)\right), \quad 1 \leq i \leq 20, \\
& =-\frac{p^{(i-20)}}{4 \sqrt{2} \Delta}\left(1+g_{t t}\right) \sinh \beta\left(\cosh \alpha+\cosh \beta-g_{t t}(\cosh \alpha-\cosh \beta)\right), \quad 21 \leq i \leq 24,
\end{aligned}
$$

whereas the spatial components of the gauge fields are given by

$$
\begin{aligned}
A_{\varphi_{k}}^{(i)}= & \frac{n^{(i)} g_{t \varphi_{k}}}{2 \sqrt{2} g_{t t}} \sinh \alpha\left(1-\frac{\left(1+g_{t t}\right)}{4 \Delta}(\cosh \alpha+\cosh \beta)\left(\cosh \alpha+\cosh \beta+g_{t t}(\cosh \alpha-\cosh \beta)\right)\right), \\
& =\frac{p^{(i-20)} g_{t \varphi_{k}}}{2 \sqrt{2} g_{t t}} \sinh \beta\left(1-\frac{\left(1+g_{t t}\right)}{4 \Delta}(\cosh \alpha+\cosh \beta)\left(\cosh \alpha+\cosh \beta-g_{t t}(\cosh \alpha-\cosh \beta)\right)\right) \\
& 21 \leq i \leq 24,
\end{aligned}
$$

with $k=1,2$. Here $\alpha$ and $\beta$ are two boost angles, $\vec{n}$ is a 20-dimensional unit vector, and $\vec{p}$ is a 4-dimensional unit vector. The nonvanishing components of the two-form field $B_{\mu \nu}$ are

$B_{t \varphi_{k}}=\frac{g_{t \varphi_{k}}}{2 g_{t t}}(\cosh \alpha-\cosh \beta)\left(1-\frac{\left(1+g_{t t}\right)}{4 \Delta}\left(\left(\sinh ^{2} \alpha+\sinh ^{2} \beta\right) g_{t t}+(\cosh \alpha+\cosh \beta)^{2}\right)\right)$

The result for the matrix-valued scalar $M$ is

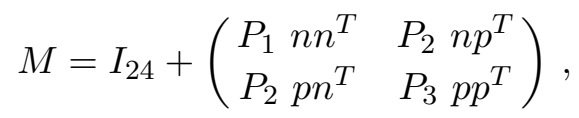

where

$$
\begin{aligned}
& P_{1}=\frac{\sinh ^{2} \alpha\left(1+g_{t t}\right)^{2}}{2 g_{t t}}\left(1-\frac{1}{4 \Delta}\left(\cosh \alpha+\cosh \beta+g_{t t}(\cosh \alpha-\cosh \beta)\right)^{2}\right), \\
& P_{2}=-\frac{2 \sinh \alpha \sinh \beta\left(1+g_{t t}\right)}{4 \Delta}\left(1+\cosh \alpha \cosh \beta+g_{t t}(\cosh \alpha \cosh \beta-1)\right), \\
& P_{3}=\frac{\sinh ^{2} \beta\left(1+g_{t t}\right)^{2}}{2 g_{t t}}\left(1-\frac{1}{4 \Delta}\left(\cosh \alpha+\cosh \beta-g_{t t}(\cosh \alpha-\cosh \beta)\right)^{2}\right) .
\end{aligned}
$$


Both black hole and black string charged rotating solutions can be generated in this way. The results in [41] are recovered for the case of a $D=6$ Myers-Perry black hole solution with only one nonzero angular momenta.

Charged rotating NUBS strings with two equal angular momenta are found by replacing in (5.7) - (5.10) the seed metric $d s^{2}$ as given by (2.2) with $g_{t t}=e^{2 G} r^{2} W^{2}-e^{2 A}\left(1-r_{0}^{2} / r^{2}\right), \quad g_{\varphi_{1} t}=$ $-e^{2 G} r^{2} W \sin ^{2} \theta, g_{\varphi_{2} t}=-e^{2 G} r^{2} W \cos ^{2} \theta$. Here we present only the line element of the charged rotating UBS solution

$$
\begin{aligned}
d \bar{s}^{2} & =\Delta(r)^{\frac{1}{4}}\left(\frac{d r^{2}}{1-\frac{2 M}{r^{2}}+\frac{2 a^{2} M}{r^{4}}}+d z^{2}+r^{2}\left(d \theta^{2}+\sin ^{2} \theta d \varphi_{1}^{2}+\cos ^{2} \theta d \varphi_{2}^{2}\right)\right) \\
& +\Delta(r)^{-\frac{3}{4}}\left(\frac{2 a M}{r^{2}\left(1-\frac{2 M}{r^{2}}\right)}\left(\Delta(r)-\frac{M}{2 r^{2}}(\cosh \alpha+\cosh \beta)\right)\left(\sin ^{2} \theta d \varphi_{1}+\cos ^{2} \theta d \varphi_{2}\right)^{2}\right. \\
& \left.-(\cosh \alpha+\cosh \beta) \frac{2 a M}{r^{2}}\left(\sin ^{2} \theta d \varphi_{1} d t+\cos ^{2} \theta d \varphi_{2} d t\right)-\left(1-\frac{2 M}{r^{2}}\right) d t^{2}\right)
\end{aligned}
$$

where

$$
\Delta(r)=1+\frac{2 M}{r^{2}}(\cosh \alpha \cosh \beta-1)+\frac{M^{2}}{r^{4}}(\cosh \alpha-\cosh \beta)^{2} .
$$

The extremal limit is found by taking the boost parameters to infinity together with a rescaling of $M$.

The asymptotic structure of a general charged solution is similar to that of the vacuum seed configuration. The spacetime still approaches the $\mathcal{M}^{5} \times S^{1}$ background as $r \rightarrow \infty$. Also, one can see that the event horizon location of the charged solutions is unchanged. The position of the ergosurface remains the same, all fields being well defined on that hypersurface. One can also verify that no closed timelike curves are introduced in the line element (5.7) by the generation procedure.

The relevant properties of a charged solution can be derived from the corresponding vacuum seed configuration. The mass-energy $\bar{E}$ and the string tension $\overline{\mathcal{T}}$ of the charged rotating solutions are

$$
\bar{E}=\frac{1}{4}(1+3 \cosh \alpha \cosh \beta) E+\frac{1}{4}(1-\cosh \alpha \cosh \beta) \mathcal{T}_{0} L, \quad \overline{\mathcal{T}}=\mathcal{T}
$$

The charged solution possesses also two equal angular momenta $\bar{J}_{1}=\bar{J}_{2}=\bar{J}$ with

$$
\bar{J}=\frac{1}{2}(\cosh \alpha+\cosh \beta) J .
$$

and has event horizon velocities $\bar{\Omega}_{1}=\bar{\Omega}_{2}=\bar{\Omega}_{H}$ with

$$
\bar{\Omega}_{H}=\frac{2 \Omega_{H}}{\cosh \alpha+\cosh \beta} .
$$

The electric charges defined as

$$
Q_{e}^{(k)}=\frac{1}{L A_{3}} \lim _{r \rightarrow \infty} \int d z \int d A_{3} r^{3} F_{r t}^{(k)}
$$


have the following expression

$$
\begin{array}{rlrl}
Q_{e}^{(i)} & =\frac{G_{6}}{\sqrt{2} \pi}\left(\frac{3 E}{L}-\mathcal{T}\right) n^{(i)} \sinh \alpha \cosh \beta, & & 1 \leq i \leq 20 \\
& =\frac{G_{6}}{\sqrt{2} \pi}\left(\frac{3 E}{L}-\mathcal{T}\right) p^{(i-20)} \sinh \beta \cosh \alpha, & 21 \leq i \leq 24
\end{array}
$$

The new solutions have a nonzero magnetic moment,

$$
\begin{array}{rlrl}
\mu^{(i)} & =\frac{G_{6} \sqrt{2}}{\pi L} J n^{(i)} \sinh \alpha, & & 1 \leq i \leq 20, \\
& =\frac{G_{6} \sqrt{2}}{\pi L} J p^{(i-20)} \sinh \beta, & 21 \leq i \leq 24 .
\end{array}
$$

Their dilaton charge is

$$
Q_{d}=\frac{G_{6}}{2 \pi}\left(\frac{3 E}{L}-\mathcal{T}\right)(\cosh \alpha \cosh \beta-1) .
$$

The relations between the Hawking temperature $\bar{T}_{H}$ and the entropy $\bar{S}$ of the charged solutions and the corresponding quantities $T_{H}$ and $S$ of the vacuum seed solution are

$$
\bar{T}_{H}=\frac{2 T_{H}}{\cosh \alpha+\cosh \beta}, \quad \bar{S}=\frac{1}{2} S(\cosh \alpha+\cosh \beta) .
$$

One can see that the entropy increases with the increase of angular momentum. On the other hand, the temperature decreases with the increase of the angular momenta. However, the products of temperature and entropy, $\bar{T}_{H} \bar{S}$, and of horizon angular velocity and angular momentum, $\bar{\Omega}_{H} \bar{J}$, are independent of the parameters $\alpha, \beta$.

As they should, all these properties reduce to those of the neutral solution upon sending the boost parameters $\alpha, \beta$ to zero.

We conclude, that every vacuum solution is associated with a family of charged solutions, which depends on 24 free parameters. In particular, the branch of non-uniform solutions emerging from the uniform black string at the threshold unstable mode thus must persist for strings with non-zero electric charge.

\section{Further remarks. Conclusions.}

Considering rotating black strings in $D$ dimensions, we have first addressed the GL instability of MP UBSs with equal magnitude angular momenta in even spacetime dimensions, taking advantage of the enhanced symmetry of these configurations. Expanding around the UBS and solving the eigenvalue problem numerically, our results indicate that the GL instability persists for these solutions up to extremality for all even dimensions between six and fourteen. This agrees with GM correlated stability conjecture [19], since these black objects are also thermodynamically unstable in a grand canonical ensemble. It may be interesting to note that the GM conjecture was also confirmed in [20] for the case of static, magnetically charged 
black strings, the Gregory-Laflamme mode vanishing at the point where the UBS becomes thermodynamically stable (which in that case is away from extremality).

While for static vacuum black strings study of the perturbative equations in second order revealed the appearance of a critical dimension, above which the perturbative nonuniform black strings are less massive than the marginally stable uniform black string [ [4], the analogous study in the presence of rotation has yet to be achieved.

In $D=6$ we then constructed numerically rotating nonuniform black strings with equal angular momenta. These emerge from the branch of marginally stable rotating MP UBS solutions, which ranges from the static marginally stable black string to the extremal rotating marginally stable black string. Along this UBS branch, the Hawking temperature $T_{H}$ decreases monotonically, reaching zero in the extremal limit. Fixing the value of the temperature (or equivalently the temperature parameter) and decreasing the value of the horizon angular velocity from the GL value, then yields a corresponding branch of rotating nonuniform black strings.

Previously, in $D=6$ dimensions, evidence was provided that the branch of static nonuniform black strings and the branch of static caged black holes merge at a topology changing transition [9], the transition occurring at critical values of the temperature $T_{*}$, the string tension $n_{*}$, etc. The results we have found for rotating NUBS indicate that at $T_{*}$ the branches of rotating NUBS, each with fixed temperature, exhibit another critical phenomenon.

In particular, the branches of rotating NUBS at fixed temperature $T_{H}>T_{*}$ end at static NUBS solutions with finite nonuniformity and thus finite waist, where the nonuniformity of these static solutions increases as $T_{H}$ is decreasing towards $T_{*}$. In contrast, along the branches of rotating NUBS at fixed temperature $T_{H}<T_{*}$ the nonuniformity parameter $\lambda$ increases apparently without bound, while at the same time the horizon angular velocity and the angular momentum appear to approach finite values. Thus for $T_{H}<T_{*}$ we see first evidence for a topology changing transition, where - in analogy with the static case - branches of rotating nonuniform black strings and branches of rotating caged black holes are expected to merge.

We conjecture that there exists a whole branch of rotating singular topology changing solutions, labelled by their decreasing temperature, beginning with the static solution at $T_{*}$, and leading possibly up to an extremal rotating solution at $T_{H}=0$. Obtaining the respective branches of rotating caged black holes represents a major numerical challenge.

In principle, there is yet another class of black objects which may play a role in this picture. Apart from configurations with an $S^{3} \times S^{1}$ (black strings) and $S^{4}$ (black holes) topology of the event horizon, the $D=6 \mathrm{KK}$ theory possesses also vacuum uniform solutions with an event horizon of topology $S^{2} \times S^{1} \times S^{1}$, corresponding to uplifted $D=5$ black rings [14, 45]. Nonuniform solutions with an event horizon of topology $S^{2} \times S^{1} \times S^{1}$, approaching at infinity the $\mathcal{M}^{5} \times S^{1}$ background are also likely to exist. They may join the MP NUBS branch at a topology changing transition. However, for the case discussed in this paper with two equal magnitude angular momenta, we could not find any indication of this scenario. This appears to be consistent with the recent results in 445, where the general $D=5$ black ring 
solution with two angular momenta was presented. An inspection of this solution indicates that black rings with equal angular momenta must exhibit some pathologies, which may explain our result.

We remark that, as in the static case [5], we observe the backbending phenomenon for the relative tension $n$ also for branches of rotating nonuniform black strings, below some critical value of the temperature.

Our last concern was the construction of charged rotating NUBS in heterotic string theory, by adding charge to the vacuum solutions by applying solution generating $(O(26-$ $D, 1) / O(26-D)) \times(O(10-D, 1) / O(10-D))$ transformations [11. The properties of these new configurations can be derived from the corresponding vacuum solutions.

We expect that, similar to the static case [8], the solutions discussed in this paper may be relevant for the thermal phase structure of non-gravitational theories, via gauge/gravity duality.

The construction of the general rotating vacuum NUMBS with distinct angular momenta seems to represent an exceedingly difficult task. However, the case of only one nonvanishing angular momentum appears to be treatable. These solutions may be found by using similar techniques to those employed in this work and are currently under study.

Although the static higher-dimensional black holes are stable [46], their rotating counterparts need not be, at least for large rotation. Recently, the existence of an effective Kerr bound for $d>4$ rapidly rotating black holes with one nonzero angular momentum was conjectured by Emparan and Myers [32]. They showed that the geometry of the event horizon of such rapidly rotating black objects in six or higher dimensions behaves like a black membrane. Therefore the black hole becomes unstable. This instability should persist for the corresponding rotating UBS solutions, and is not associated with the extra dimensions.

Rotating black objects extending in extra dimensions may also exhibit other instabilities. Cardoso and Lemos [47] uncovered a new universal instability for rotating black branes and

strings, which holds for any massless field perturbation. The main point of their argument is that transverse dimensions in a black string geometry act as an effective mass for the fields, which simulates a mirror enclosing a rotating black hole, thereby creating a black hole bomb. For further work on the instabilities of rotating black objects, see for example [48], [49], [50].

\section{Acknowledgements}

B.K. gratefully acknowledges support by the DFG under contract KU612/9-1. The work of E.R. was carried out in the framework of Enterprise-Ireland Basic Science Research Project $\mathrm{SC} / 2003 / 390$.

\section{References}

[1] R. Gregory and R. Laflamme, Phys. Rev. Lett. 70 (1993) 2837 [arXiv:hep-th/9301052].

[2] S. S. Gubser, Class. Quant. Grav. 19 (2002) 4825 [arXiv:hep-th/0110193]. 
[3] T. Wiseman, Class. Quant. Grav. 20 (2003) 1137 [arXiv:hep-th/0209051].

[4] E. Sorkin, Phys. Rev. Lett. 93 (2004) 031601 [arXiv:hep-th/0402216].

[5] B. Kleihaus, J. Kunz and E. Radu, JHEP 0606 (2006) 016 [arXiv:hep-th/0603119].

[6] E. Sorkin, Phys. Rev. D 74 (2006) 104027 [arXiv:gr-qc/0608115].

[7] B. Kol, Phys. Rept. 422 (2006) 119 [arXiv:hep-th/0411240].

[8] T. Harmark, V. Niarchos and N. A. Obers, arXiv:hep-th/0701022.

[9] H. Kudoh and T. Wiseman, Phys. Rev. Lett. 94 (2005) 161102 [arXiv:hep-th/0409111].

[10] B. Kol, JHEP 0510 (2005) 049 [arXiv:hep-th/0206220].

[11] T. Harmark and N. A. Obers, JHEP 0409 (2004) 022 [arXiv:hep-th/0407094].

[12] T. Harmark, K. R. Kristjansson, N. A. Obers and P. B. Ronne, JHEP 0701 (2007) 023 [arXiv:hep-th/0606246].

[13] B. D. Chowdhury, S. Giusto and S. D. Mathur, Nucl. Phys. B 762 (2007) 301 [arXiv:hep-th/0610069].

[14] R. Emparan and H. S. Reall, Phys. Rev. Lett. 88 (2002) 101101 [arXiv:hep-th/0110260].

[15] R. C. Myers and M. J. Perry, Annals Phys. 172 (1986) 304.

[16] H. K. Kunduri, J. Lucietti and H. S. Reall, Phys. Rev. D 74 (2006) 084021 [arXiv:hep-th/0606076].

[17] J. Kunz, F. Navarro-Lerida and J. Viebahn, Phys. Lett. B 639 (2006) 362 [arXiv:hep-th/0605075].

[18] J. Kunz, D. Maison, F. Navarro-Lerida and J. Viebahn, Phys. Lett. B 639 (2006) 95 [arXiv:hep-th/0606005].

[19] S. S. Gubser and I. Mitra, arXiv:hep-th/0009126.;

S. S. Gubser and I. Mitra, JHEP 0108 (2001) 018 [arXiv:hep-th/0011127].

[20] U. Miyamoto and H. Kudoh, JHEP 0612 (2006) 048 [arXiv:gr-qc/0609046].

[21] H. Kudoh and U. Miyamoto, Class. Quant. Grav. 22 (2005) 3853 [arXiv:hep-th/0506019].

[22] G. W. Gibbons and S. W. Hawking, Phys. Rev. D 15 (1977) 2752.

[23] P. K. Townsend and M. Zamaklar, Class. Quant. Grav. 18 (2001) 5269 [arXiv:hep-th/0107228].

[24] J. D. Brown and J. W. York, Phys. Rev. D 47 (1993) 1407.

[25] P. Kraus, F. Larsen and R. Siebelink, Nucl. Phys. B 563 (1999) 259 [arXiv:hep-th/9906127];

[26] D. Astefanesei, R. B. Mann and C. Stelea, arXiv:hep-th/0608037.

[27] R. B. Mann and D. Marolf, Class. Quant. Grav. 23 (2006) 2927 [arXiv:hep-th/0511096].

[28] D. Astefanesei and E. Radu, Phys. Rev. D 73 (2006) 044014 [arXiv:hep-th/0509144].

[29] B. Kol, E. Sorkin and T. Piran, Phys. Rev. D 69 (2004) 064031 [arXiv:hep-th/0309190].

[30] G. T. Horowitz, arXiv:hep-th/0205069. 
[31] T. Harmark and N. A. Obers, Nucl. Phys. B 684 (2004) 183 [arXiv:hep-th/0309230].

[32] R. Emparan and R. C. Myers, JHEP 0309 (2003) 025 [arXiv:hep-th/0308056].

[33] R. B. Mann, Found. Phys. 33 (2003) 65 [arXiv:gr-qc/0211047].

[34] M. Cvetic and S. S. Gubser, JHEP 9904 (1999) 024 [arXiv:hep-th/9902195].

[35] M. M. Caldarelli, G. Cognola and D. Klemm, Class. Quant. Grav. 17 (2000) 399 [arXiv:hep-th/9908022].

[36] W. Schönauer and R. Weiß, J. Comput. Appl. Math. 27, 279 (1989) 279;

M. Schauder, R. Weiß and W. Schönauer, The CADSOL Program Package, Universität Karlsruhe, Interner Bericht Nr. 46/92 (1992).

[37] B. Kleihaus and J. Kunz, Phys. Rev. D 57 (1998) 834 [arXiv:gr-qc/9707045];

B. Kleihaus and J. Kunz, Phys. Rev. D 57 (1998) 6138 [arXiv:gr-qc/9712086];

B. Kleihaus, J. Kunz and F. Navarro-Lerida, Phys. Rev. D 66 (2002) 104001 [arXiv:gr-qc/0207042].

[38] B. Kol and T. Wiseman, Class. Quant. Grav. 20 (2003) 3493 [arXiv:hep-th/0304070].

[39] T. Wiseman, Class. Quant. Grav. 20 (2003) 1177 [arXiv:hep-th/0211028].

[40] J. Maharana and J. H. Schwarz, Nucl. Phys. B 390 (1993) 3 [arXiv:hep-th/9207016].

[41] A. Sen, Nucl. Phys. B 440 (1995) 421 [arXiv:hep-th/9411187].

[42] A. W. Peet, Nucl. Phys. B 456 (1995) 732 [arXiv:hep-th/9506200].

[43] G. T. Horowitz and A. Sen, Phys. Rev. D 53 (1996) 808 [arXiv:hep-th/9509108].

[44] M. Cvetic and D. Youm, Nucl. Phys. B 477 (1996) 449 [arXiv:hep-th/9605051].

[45] A. A. Pomeransky and R. A. Sen'kov, arXiv:hep-th/0612005.

[46] A. Ishibashi and H. Kodama, Prog. Theor. Phys. 110 (2003) 901 [arXiv:hep-th/0305185]; H. Kodama and A. Ishibashi, Prog. Theor. Phys. 111 (2004) 29 [arXiv:hep-th/0308128].

[47] V. Cardoso and J. P. S. Lemos, Phys. Lett. B 621 (2005) 219 [arXiv:hep-th/0412078].

[48] G. Gibbons and S. A. Hartnoll, Phys. Rev. D 66 (2002) 064024 [arXiv:hep-th/0206202].

[49] D. Marolf and B. C. Palmer, Phys. Rev. D 70 (2004) 084045 [arXiv:hep-th/0404139].

[50] V. Cardoso and O. J. C. Dias, Phys. Rev. Lett. 96 (2006) 181601 [arXiv:hep-th/0602017]; V. Cardoso and L. Gualtieri, Class. Quant. Grav. 23 (2006) 7151 [arXiv:hep-th/0610004]. 